\title{
EPSILON ERIDANI'S PLANETARY DEBRIS DISK: STRUCTURE AND DYNAMICS BASED ON SPITZER AND CALTECH SUBMILLIMETER OBSERVATORY OBSERVATIONS
}

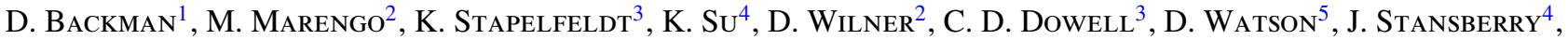 \\ G. RIEKE ${ }^{4}$, T. MEGEATH ${ }^{2,6}$, G. FAZIO ${ }^{2}$, AND M. WERNER ${ }^{3}$ \\ ${ }^{1}$ Stratospheric Observatory for Infrared Astronomy \& SETI Institute, 515 North Whisman Road, Mountain View, CA 94043, USA \\ ${ }^{2}$ Harvard-Smithsonian Center for Astrophysics, 60 Garden Street, Cambridge, MA 02138, USA \\ ${ }^{3}$ Jet Propulsion Laboratory, California Institute of Technology, 4800 Oak Grove Drive, Pasadena, CA 91109, USA \\ ${ }^{4}$ Steward Observatory, University of Arizona, Tucson, AZ 85721, USA \\ ${ }^{5}$ Department of Physics and Astronomy, University of Rochester, Rochester, NY 14627, USA \\ Received 2008 May 25; accepted 2008 September 8; published 2008 December 22
}

\begin{abstract}
Spitzer and Caltech Submillimeter Observatory images and spectrophotometry of $\epsilon$ Eridani at wavelengths from 3.5 to $350 \mu \mathrm{m}$ reveal new details of its bright debris disk. The $350 \mu \mathrm{m}$ map confirms the presence of a ring at $r=11^{\prime \prime}-28^{\prime \prime}(35-90 \mathrm{AU})$, observed previously at longer sub-mm wavelengths. The Spitzer mid-IR and farIR images do not show the ring, but rather a featureless disk extending from within a few arcsec of the star across the ring to $r \sim 34^{\prime \prime}(110 \mathrm{AU})$. The spectral energy distribution (SED) of the debris system implies a complex structure. A model constrained by the surface brightness profiles and the SED indicates that the sub-mm ring emission is primarily from large $(a \sim 135 \mu \mathrm{m})$ grains, with smaller $(a \sim 15 \mu \mathrm{m})$ grains also present in and beyond the ring. The Spitzer Infrared Spectrograph and Multiband Imaging Photometer for Spitzer SEDmode spectrophotometry data clearly show the presence of spatially compact excess emission at $\lambda \gtrsim 15 \mu \mathrm{m}$ that requires the presence of two additional narrow belts of dust within the sub-mm ring's central void. The innermost belt at $r \sim 3 \mathrm{AU}$ is composed of silicate dust. A simple dynamical model suggests that dust produced collisionally by a population of about $11 M_{\oplus}$ of planetesimals in the sub-mm ring could be the source of the emission from both in and beyond the sub-mm ring. Maintaining the inner belts and the inner edge to the sub$\mathrm{mm}$ ring may require the presence of three planets in this system including the candidate radial velocity object.
\end{abstract}

Key words: circumstellar matter - infrared: stars - planetary systems - stars: individual (epsilon Eridani)

Online-only material: color figure

\section{INTRODUCTION}

The very nearby (3.22 pc; HIP 16537) K2 V star $\epsilon$ Eridani (HR 1084, HD 22049, GJ 144) was found by Infrared Astronomical Satellite (IRAS) to have a bright semiresolved far-IR (FIR) excess due to circumstellar dust (Gillett 1984; Aumann 1985). The system shows $\geqslant 1$ Jy of excess at 60 $200 \mu \mathrm{m}$ (IRAS 03305-0937; Walker \& Heinrichsen 2000 and references therein) and a moderate-significance excess at $25 \mu \mathrm{m}$.

Very Large Telescope Interferometer (VLTI) angular diameter measurements plus evolutionary models imply an age for $\epsilon$ Eri of 850 Myr (Di Folco et al. 2004), consistent with previous determinations based on Ca II activity (Henry et al. 1996), Li abundances (Song et al. 2000), and gyrochronology (Barnes 2007). For $\epsilon$ Eri, as well as the other IRAS-discovered prototype debris disk systems $\alpha$ Lyr (Vega), $\alpha$ PsA (Fomalhaut), and $\beta$ Pic, (Gillett 1984, 1986), later dubbed the "Fab Four," estimated timescales for dust destruction by Poynting-Robertson $(\mathrm{P}-\mathrm{R})$ drag and mutual collisions are significantly less than the respective system ages. Thus, the observed dust is not primordial but must be "second-generation" material released relatively recently, by collisions or other activity, from larger parent bodies. These debris disks are, therefore, understood to represent late stages, or remnants, of the planet-formation process. Many more debris disks have been discovered since the prototypes (see reviews by Backman \& Paresce 1993, Protostars and Planets III; Lagrange et al. 2000, Protostars and Planets IV; Meyer et al.

\footnotetext{
$\overline{6}$ Now at the University of Toledo, OH, USA.
}

2007, Protostars and Planets V). $\epsilon$ Eri is especially interesting for its proximity, its age of nearly $1 \mathrm{Gyr}$, and its $\mathrm{K}$ main-sequence spectral type for which relatively few debris disks are presently known (Trilling et al. 2008).

The $\epsilon$ Eri system was mapped at 850 and $450 \mu \mathrm{m}$ by Greaves et al. $(1998,2005)$, revealing a nearly circular ring extending more than $25^{\prime \prime}$ (80 AU) from the star, with peak surface brightness at about $18^{\prime \prime}(\sim 60 \mathrm{AU})$ and a central cavity with radius about $10^{\prime \prime}(\sim 30 \mathrm{AU})$. Schütz et al. (2004) detected the ring at $1.2 \mathrm{~mm}$ and confirmed its spatial extent. Marengo et al. (2006) made a deep search at wavelengths of 3.5-7.9 $\mu \mathrm{m}$ for planetary-mass companions between $14^{\prime \prime}(45 \mathrm{AU})$ and 2.9 (560 AU) from the star with Spitzer's Infrared Array Camera (IRAC), and also set upper limits to extended emission. Proffitt et al. (2004) used Hubble Space Telescope/Space Telescope Imaging Spectrograph (HST/STIS) observations to determine an upper limit to optical scattered light from the region of the sub-mm ring. Di Folco et al. (2007) set an upper limit on nearIR (NIR; $K^{\prime}$-band) scattered light brightness within $1^{\prime \prime}(3 \mathrm{AU})$ of $\epsilon$ Eri via Center for High Angular Resolution Astronomy (CHARA) interferometric measurements.

Dent et al. (2000), Li et al. (2003), and Sheret et al. (2004) calculated disk models constrained by the surface brightness distribution in the sub-mm images and pre-Spitzer spectral energy distribution (SED) data, examining alternatives of porous or solid grain structures and silicate or silicate-plus-ice compositions. Bright spots in the sub-mm ring have been interpreted as dust density enhancements caused by a planet orbiting in or near the ring (Liou \& Zook 1999; Ozernoy et al. 2000; Quillen 
\& Thorndike 2002; Deller \& Maddison 2005; Greaves et al. 2005).

Tentative detection of a Jovian-mass planet orbiting $\epsilon$ Eri with a semimajor axis of $3.4 \mathrm{AU}$, well inside the sub-mm ring, has been made via radial velocity measurements (Campbell et al. 1988; Hatzes et al. 2000), possibly confirmed astrometrically (Benedict et al. 2006). Das \& Backman (1992) suggested that such a planet might influence the location of the warm dust detected by IRAS. Detailed models of the gravitational effect of a planet close to the star on possible nearby dust distributions were calculated by Moran et al. (2004).

The present paper is part of the Spitzer all-instrument Guaranteed Time Observing (GTO) "Fab Four" program, which makes comprehensive studies of the prototype planetary debris disks to put our solar system into evolutionary context and to search for signs of planets (Fomalhaut: Stapelfeldt et al. 2004; Vega: Su et al. 2005; $\beta$ Pic: Chen et al. 2007). Spitzer obtained the first FIR images of $\epsilon$ Eri, which yield broadband photometry and disk surface brightness profiles as well as high-resolution comparisons with prior sub-mm maps. These Spitzer images are combined here with medium-resolution IR spectroscopy plus a $350 \mu \mathrm{m}$ map from the Caltech Submillimeter Observatory (CSO) SHARC II camera. Together, these data provide a unique opportunity to study the nearest-known exo-Kuiper belt and exo-zodiacal dust system in detail.

Section 2 of this paper describes the Spitzer and CSO observations of $\epsilon$ Eri and the data reduction. Section 3 addresses detailed analyses of the data including first-order corrections of image and spectrophotometric data plus determination of the photosphere SED and IR excess. Section 4 describes derivation of a model of grain spatial and size distributions fitted to the disk's multi- $\lambda$ surface brightness distributions and overall SED. Section 5 discusses implications of the model results, compares them with other observations and SED-based models of the $\epsilon$ Eri disk, and presents results of a simple dynamical model calculation connecting $\epsilon$ Eri's disk properties with the history of our solar system's Kuiper belt.

\section{OBSERVATIONS AND DATA REDUCTION}

Spitzer observations of $\epsilon$ Eri were taken in GTO Program 90 (P.I. Michael Werner). Data obtained with various instruments and facilities were used as follows. (1) Spitzer Infrared Array Camera (IRAC) image photometry measures the photospheric SED from 3.6 to $7.9 \mu \mathrm{m}$, wavelengths that show no detectable contribution from the debris disk. (2) Multiband Imaging Photometer (MIPS) for Spitzer data at 24, 70, and $160 \mu \mathrm{m}$ provide mid-IR and FIR images of the disk. (3) Spitzer MIPS SED-mode data give source flux densities from 55 to $90 \mu \mathrm{m}$ with low spectral resolution, plus source size in one dimension versus wavelength. (4) Spitzer Infrared Spectrograph (IRS) spectra link the mid-IR broadband photometric points, determine the shortest wavelength of excess (highest abundant dust temperature, minimum dust orbit radius), and allow a search for mineralogical features. (5) The CSO $350 \mu \mathrm{m}$ map can be compared with the Spitzer FIR images as well as with prior 450 and $850 \mu \mathrm{m}$ maps. Surface brightness profiles from the $24,70,160$, and $350 \mu \mathrm{m}$ data together strongly constrain the disk model. (6) The Spitzer MIPS images and CSO map are also integrated to yield broadband photometric points for the entire disk, supplemented by IRAS catalog photometry using recalculated color corrections plus previously-published ISO photometry.

\subsection{Spitzer IRAC Data}

Images at 3.6-7.9 $\mu \mathrm{m}$ of $\epsilon$ Eri and its surroundings were made with Spitzer's IRAC (Fazio et al. 2004) in 2004 January and February. These observations were designed to look for wideseparation low-mass companions, but also provided accurate photometry of the star via point-spread function (PSF) fitting of nonsaturated portions of $\epsilon$ Eri and standard star images. Details of the observations and data reduction procedures are described by Marengo et al. (2006). The IRAC data were reprocessed for the present paper to take advantage of an updated instrument calibration plus availability of additional reference star observations to generate a new PSF.

Each of the two IRAC observations consisted of a 36-position dither of the source on the array, for a total of $2 \times 3380 \mathrm{~s}$ integration time. The new PSF reference stars were $\epsilon$ Indi (K4.5 V), Vega, Fomalhaut, and Sirius. The debris disks around Vega and Fomalhaut are not detected at IRAC wavelengths. The total field of view (FOV) had a width of 5.78 due to the dither, slightly larger than the IRAC frame width of 5.21. Data reduction and calibration were done using the Spitzer Science Center (SSC) pipeline version S15 combined with the postBasic Calibrated Data (BCD) IRACproc software (Schuster et al. 2006).

\subsection{Spitzer MIPS Data}

\subsubsection{MIPS 24, 70, and $160 \mu \mathrm{m}$ Images}

Observations of $\epsilon$ Eri were made using all three imaging channels of the MIPS (Rieke et al. 2004). Images at $24 \mu \mathrm{m}$ were made on 2004 January 29 using two different observing strategies. (1) The standard $24 \mu \mathrm{m}$ MIPS photometry dither pattern was executed at four cluster target positions in a square pattern 8.'29 (3.25 pixels) wide. This procedure yielded an exposure time of $4 \times(16 \times 3) \mathrm{s}$ for the region within 2.5 of the star, with a high degree of subpixel sampling to facilitate PSF subtraction. Observations of the K4 III star HD 217382 made on 2003 November 19 with the identical dither/cluster target pattern were used as a PSF reference. (2) To probe a wider FOV for possible companions, another $\epsilon$ Eri $24 \mu \mathrm{m}$ dataset was taken using a $3 \times 3$ raster map. The resulting mosaic covers a $0.25 \times 0.25$ region centered on the star to an exposure depth of $2 \times(16 \times 3) \mathrm{s}$.

Two types of MIPS $70 \mu \mathrm{m}$ image observations were also taken on 2004 January 29. (1) With the default pixel scale ( 9 '.96 per pixel), eight cycles of the large-field photometry dither pattern were used, providing a total exposure time of $151 \mathrm{~s}$ per pixel. (2) With the fine pixel scale (5'.2 per pixel), one cycle of the small-field photometry dither pattern was made at each of 12 cluster target positions, arranged in a rectangular grid with 16 '.22 (3.25 pixel) spacing, for an effective exposure time of $12 \times(10 \times 3)$ s per pixel. On 2005 April 4 a deeper finescale image was obtained with an effective exposure time of $12 \times(10 \times 10)$ s per pixel.

Two MIPS $160 \mu \mathrm{m}$ image datasets were obtained. (1) The first set was taken on 2004 January 29, using two cycles of small-field photometry at each of nine cluster target positions spaced on a $36^{\prime \prime}$ rectangular grid, and $3 \mathrm{~s}$ exposures, for an exposure time of $9 \times 27 \mathrm{~s}$ per pixel. (2) Another set was obtained on 2006 February 19 using the same observing strategy except that $10 \mathrm{~s}$ exposures were used, for an exposure time of $9 \times 90 \mathrm{~s}$.

The MIPS $160 \mu \mathrm{m}$ camera suffers from a spectral leak by which stray light in the wavelength range $1-1.6 \mu \mathrm{m}$ produces a false image partially overlapping the actual $160 \mu \mathrm{m}$ source 
image. This ghost image is several times brighter than the real disk emission in the case of $\epsilon$ Eri, and must be accurately subtracted to enable the study of the circumstellar emission. The spectral leak is field-dependent, and, therefore, can only be properly subtracted using a reference star that was observed with the same dither parameters as the science target. Prelaunch observation planning did not anticipate this requirement, so no appropriate reference star observation was initially available to leak-subtract the $\epsilon$ Eri dataset.

Good leak subtraction was achieved using $160 \mu \mathrm{m}$ images taken on 2004 August 6 of the debris disk star $\tau$ Ceti (type G8) with the same dither pattern as for $\epsilon$ Eri. Only the spectral leak, and not the photosphere or disk excess, is detected at $160 \mu \mathrm{m}$ from $\tau$ Ceti (K. Stapelfeldt et al. 2009, in preparation). The subtraction procedure involved empirically determining the maximum normalization factor for the leak reference source such that its subtraction from the science target did not produce noticeable residuals below the background level. The subtractions were performed using mosaics in detector coordinates of all the dithered observations. Observations of Achernar ( $\alpha$ Eri) were also obtained for $\epsilon$ Eri leak subtraction reference, on 2005 August 30. The Achernar data did not support a good leak subtraction for $\epsilon$ Eri, probably because of the large color difference between the two stars (type B3 versus $\mathrm{K} 2$ ).

The MIPS instrument team's Data Analysis Tool (DAT; Gordon et al. 2005) was used for basic reduction (dark subtraction, flat fielding/illumination correction) of the SSC S11 data products. The $24 \mu \mathrm{m}$ images were processed to remove a vertical "jailbar" pattern along the detector columns that appears during the observations of bright sources. The known transient behaviors associated with the MIPS $70 \mu \mathrm{m}$ array were removed from the default-scale data by median column subtraction and time-filtering the images with an excluded region of 15 pixels $\left(\sim 150^{\prime \prime}\right)$ centered on the source (for details, see Gordon et al. 2007). For the $70 \mu \mathrm{m}$ fine-scale data, off-source exposures were subtracted from preceding on-source exposures to remove the time-dependent background column offsets. Flux calibration factors of $0.0454 \mathrm{MJy} \mathrm{sr}^{-1}(24 \mu \mathrm{m}), 702 \mathrm{MJy} \mathrm{sr}^{-1}$ (70 $\mu \mathrm{m}$ default-scale), $2894 \mathrm{MJy} \mathrm{sr}^{-1}$ (70 $\mu \mathrm{m}$ fine-scale), and

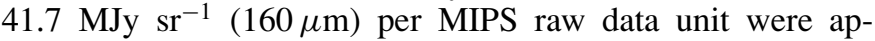
plied to the MIPS image data (Engelbracht et al. 2007; Gordon et al. 2007; Stansberry et al. 2007). The processed data were then combined using World Coordinate System (WCS) information to produce final mosaics with pixels half the size of the physical pixel scale.

\subsubsection{MIPS SED-Mode Data}

MIPS SED-mode data were obtained on 2005 September 4 using ten cycles of $10 \mathrm{~s}$ exposures with a $+3^{\prime}$ chopper throw. The total integration time was $629 \mathrm{~s}$. Basic reduction of the data to produce calibrated versions of the individual exposures was done using version 3.06 of the MIPS DAT code. MIPS SED-mode observations provide chopped pairs of images. The off-source images were pair-wise subtracted from the on-source images, and the results mosaicked to produce an image of the 55-90 $\mu \mathrm{m}$ spectrum of $\epsilon$ Eri. The mosaic was generated using 4 .'9 pixels, yielding an image 65 pixels across in the spectral dimension by 44 pixels in the spatial dimension. This image was then boxcar-smoothed over five adjacent rows, resulting in spectral resolution $R=15-20$.

\subsection{Spitzer IRS Data}

Mid-IR spectra of $\epsilon$ Eri were obtained in 2004 February, 2004 August, and 2005 January with the Spitzer IRS (Houck et al. 2004). Using the low-spectral resolution $(R \sim 60-120)$ spectrographs SL1 $(7.5-14 \mu \mathrm{m})$, LL2 (14-21 $\mu \mathrm{m})$, and LL1 $(20-38 \mu \mathrm{m})$, observations were made with five slit positions separated by half a slit width (1".8) in SL1 and ten slit positions separated by half a slit width (4".8) in LL2 and LL1. The star was centered in the scan pattern in each case. Staring-mode observations were also obtained using the low-resolution SL2 $(5.3-7.5 \mu \mathrm{m})$ and high resolution $(R \sim 600) \mathrm{SH}(10-20 \mu \mathrm{m})$ and LH (19-37 $\mu \mathrm{m})$ modules. These observations were at two nod positions, with the star displaced from the center of the slit by a third of the slit length. Total integration times per slit position or nod were $84 \mathrm{~s}$ in the SL1 spectral map, $70 \mathrm{~s}$ each in the LL2 and LL1 spectral maps, and 54 s, 72 s, and 144 s, for the SL2, SH, and LH observations, respectively. Each set of observations was preceded by a high-accuracy pointing peak-up observation of the neighboring star SAO 130582 using the IRS Blue $(15 \mu \mathrm{m})$ camera.

Data reduction began with products of the SSC's S11 IRS data pipeline. Further processing involved removal, by interpolation in the spectral direction, of permanently bad and "rogue" pixels identified in the IRS dark-current data for all campaigns up to and including the one in which each $\epsilon$ Eri spectrum was taken. The IRS instrument team's SMART software was then used to extract one-dimensional spectra from the two-dimensional long-slit (SL, LL) or echelle (SH, LH) images. Sky emission in the SL and LL observations was measured by a linear fit to the signals at points along the slit that are farther than $40^{\prime \prime}$ from the star, and subtracted as part of the extraction. The $\epsilon$ Eri system appeared to be spatially unresolved in the SL observations, so an extraction window was used that was fitted to the instrumental PSF at the longest wavelengths of each low-resolution module, 4 pixels wide in each case. A full-slit extraction was performed for the high-spectral-resolution ( $\mathrm{SH}$ and $\mathrm{LH}$ ) observations. The sky emission measured in the low-spectral-resolution observations was small enough, compared with the flux received from $\epsilon$ Eri and its disk, so that sky subtraction in the $\mathrm{SH}$ and $\mathrm{LH}$ observations could be neglected.

Reference SL and LL observations of $\alpha$ Lac (A1 V) plus SH and LH observations of $\xi$ Dra (K1 III) were obtained in the same manner as the $\epsilon$ Eri observations. The spectra of $\epsilon$ Eri were calibrated via division by spectra of the respective reference star taken at the corresponding nod position, multiplication by a synthetic template spectrum of the reference star (M. Cohen 2003, private communication), and then nod-averaging. Reduced spectra from each spectrograph module were then combined to produce complete 5.3-38 $\mu \mathrm{m}$ spectra. Small spectrograph module-to-module differences at wavelengths of overlap were assumed to be due to minor telescope pointing-induced throughput losses, and removed by scaling up the spectra from smaller-slit modules to match those from the larger-slit modules in the overlap range.

Linearity and saturation in the IRS detector arrays are corrected in the data pipeline, but $\epsilon$ Eri and its disk are bright enough such that linearity and saturation effects in the low spectral resolution modules are large and the corrections uncertain. The main impact of these effects is that the LL spectra had a significantly lower effective signal-to-noise ratio $(\mathrm{S} / \mathrm{N})$ and spectrophotometric accuracy than the SH and $\mathrm{LH}$ observations due to residual inaccuracy of the LL saturation correction. The final spectrum used in this paper was assembled 


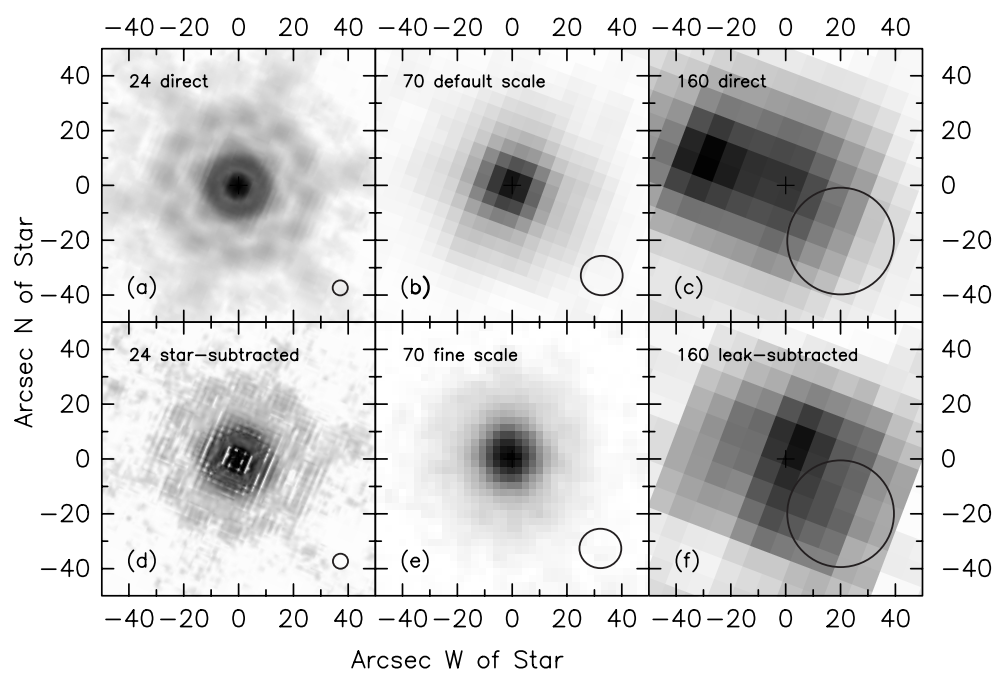

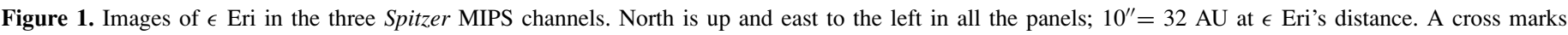

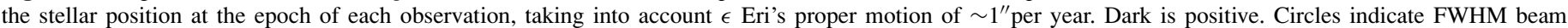

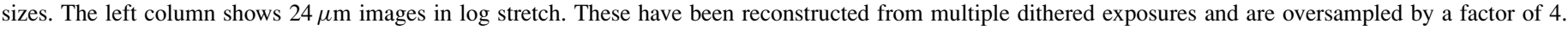

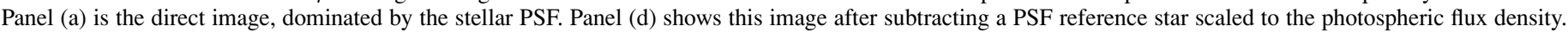

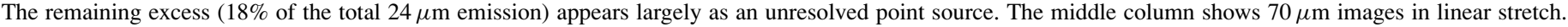

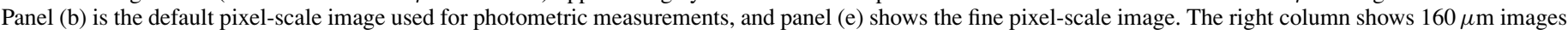
in linear stretch. Panel (c) is the direct image and panel (f) shows this image after subtraction of the ghost image arising from the spectral leak.

from SL, SH, and LH data; LL data will not be discussed further. The uncertainty in flux density is $5 \%$ below about $10 \mathrm{Jy}$, increasing to about $10 \%$ for flux densities as high as $20 \mathrm{Jy}$.

\subsection{CSO Data}

Observations of $\epsilon$ Eri at $350 \mu \mathrm{m}$ were made during parts of three nights in 2003 January and two nights in 2005 December using the SHARC II $12 \times 32$ bolometer array (Dowell et al. 2003) at the CSO. The weather conditions were excellent, with $225 \mathrm{GHz}(1.3 \mathrm{~mm})$ zenith opacity ranging from 0.028 to 0.048 . The total integration time on the source was $11 \mathrm{hr}$. Data were obtained by rapid scanning (without chopping) in Lissajous patterns with periods of 14-20 s and amplitudes of $\pm 15^{\prime \prime}$ to $\pm 40^{\prime \prime}$ in each of two axes.

Each SHARC II detector spans 4."6, and the diffractionlimited beam size is $9^{\prime \prime}(\mathrm{FWHM})$, with a PSF determined by coadding images from a variety of calibration sources interspersed with the $\epsilon$ Eri observations. Data reduction used the sharcsolve software package that iteratively decomposes fixed source emission, time-variable atmospheric emission, and detector offsets. Best results for subtraction of the temporally and spatially variable telluric emission were achieved by assuming that the average emission outside a $35^{\prime \prime}$ radius from the star was zero, at the cost of filtering out possible more-widely extended emission. Calibration was based on the integrated map signals within a $35^{\prime \prime}$ radius, following correction for atmospheric absorption, from sub-mm standards OH231.8 (18 Jy), CRL618 (19 Jy), o Ceti (2.3 Jy), Ceres (13 Jy in 2003 January), and Juno (8.0 Jy in 2005 December). The uncertainly in the absolute flux scale is estimated to be $25 \%$, and the root mean square (rms) noise level in the reduced $350 \mu \mathrm{m}$ image is $\sim 3 \mathrm{mJy}$ per smoothed beam.

\section{RESULTS}

\subsection{Images and Spatial Structure}

\subsubsection{MIPS FIR Images}

The top row of Figure 1 displays the MIPS 24, 70, and $160 \mu \mathrm{m}$ images after standard Spitzer pipeline processing. The bottom row of Figure 1 shows the output from a further level of processing. Specifically, (1) Figure 1(d) is the result of subtracting a scaled PSF from the $24 \mu \mathrm{m}$ image in Figure 1(a), (2) Figure 1(e) shows a $70 \mu \mathrm{m}$ fine pixel-scale image for comparison with the default pixel-scale image in Figure 1(b), and (3) Figure 1(f) is the result of compensating for the $160 \mu \mathrm{m}$ filter's short-wavelength leak that is apparent in Figure 1(c). Radial profiles of the processed images are presented and discussed in Section 4.2.1.

The $24 \mu \mathrm{m}$ PSF-subtracted data show an image core size of $5^{\prime \prime} 6 \times 5$ ". 4 , consistent with the values of 5".52 $\times 5$ ".48 for an unresolved blue (photospheric) source determined by Su et al. (2008). The stripes in the processed data are PSF subtraction residuals, mainly noise arising from different sampling of the source and PSF reference by the pixel grid. Examination of the $24 \mu \mathrm{m}$ PSF wings shows evidence for slight excess surface brightness at the position of the first Airy dark ring $\left(r \sim 7^{\prime \prime}\right)$ but with only $\sim 1 \sigma$ significance.

The FIR source is resolved at 70 and $160 \mu \mathrm{m}$. At $70 \mu \mathrm{m}$ (fine pixel-scale image), the observed source has a compact core with a FWHM of 24 ". $2 \times 22$ ". 3 elongated approximately north-south, superimposed on a relatively flat pedestal of emission roughly $1^{\prime}$ in diameter. The core $70 \mu \mathrm{m}$ emission lies mostly interior to the sub-mm ring, and is clearly extended relative to the size of unresolved point sources, which is $15^{\prime \prime} .3 \times 14^{\prime \prime} .4$ (Su et al. 2008). The leak-subtracted $160 \mu \mathrm{m}$ source can be approximated by Gaussian profiles with FWHM $62^{\prime \prime} \times 52^{\prime \prime}$, also elongated north-south. This distribution is also extended relative to a point source (39"diffraction FWHM). The stellar point source contributes about $10 \%$ of the total system flux density at $70 \mu \mathrm{m}$ and $5 \%$ at $160 \mu \mathrm{m}$.

The 70 and $160 \mu \mathrm{m}$ images contain a substantial surprise. Although the FIR source FWHM is about the size of the sub$\mathrm{mm}$ ring, the FIR images do not show the ring-with-deep-hole aspect observed at longer wavelengths. Instead, the FIR surface brightness distribution is continuous across the central region and the sub-mm ring, extending well beyond the ring, with no significant enhancement at the position of the ring. The FIR source is close to circularly symmetric, with a possible slight 


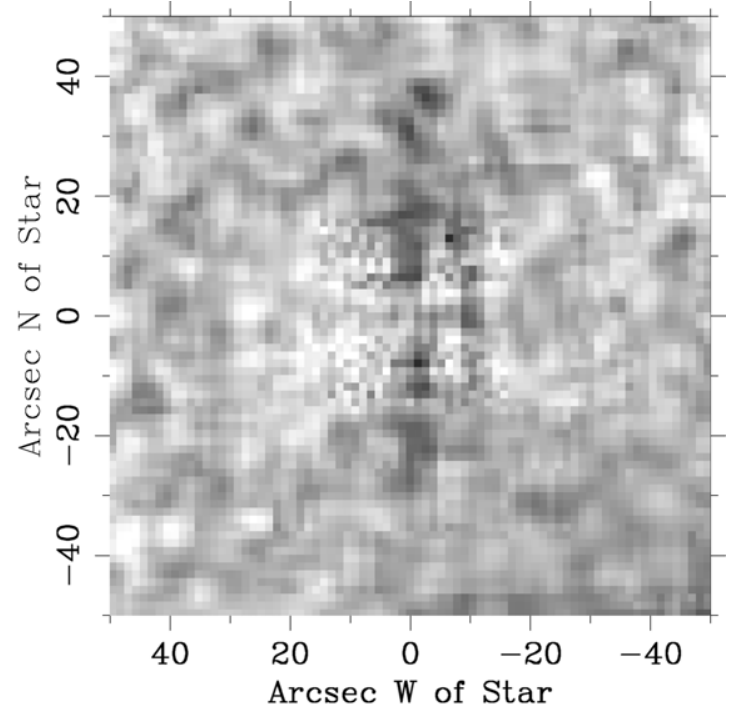

(a)

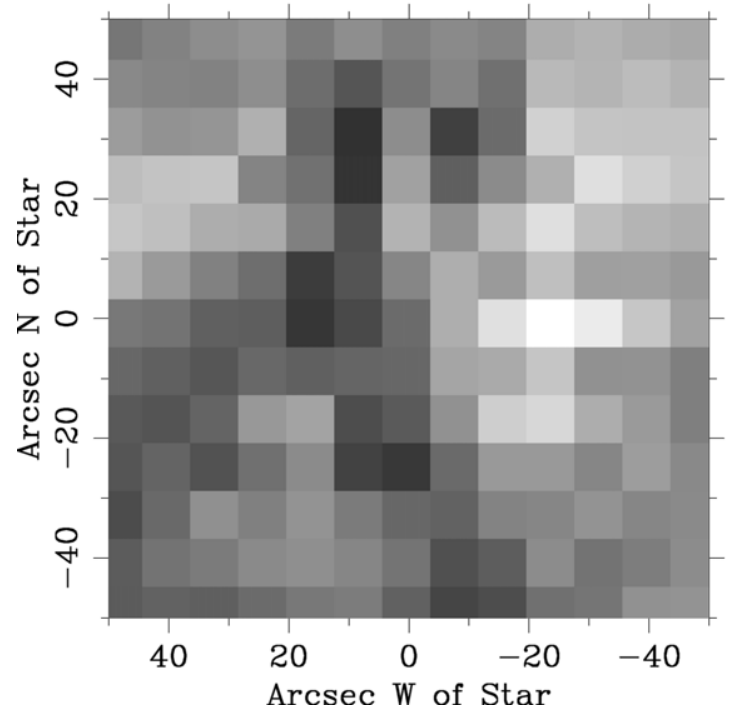

(b)

Figure 2. (a) Difference between the Spitzer MIPS fine-scale $70 \mu \mathrm{m}$ image and its azimuthal average. (b) The same for the MIPS $160 \mu \mathrm{m}$ image. The vertical bars are due to incomplete correction of column artifacts. The remaining residuals are consistent with noise from the subtraction, indicating the absence of surface brightness features in the sub-mm ring within the sensitivity limits.

north-south extension as in the 350 and $450 \mu \mathrm{m}$ source maps, but unlike the $850 \mu \mathrm{m}$ maps that are extended east-west or northeastsouthwest (Greaves et al. 2005).

To test for FIR detection of surface brightness features seen in the sub-mm ring, an azimuthal average of the $70 \mu \mathrm{m}$ finescale image was subtracted from the image itself. Figure 2(a) shows the result of this analysis. The subtracted image is smooth to $\lesssim 20 \%$ of the surface brightness in the ring, smaller than the amplitude of features in prior sub-mm maps. The $70 \mu \mathrm{m}$ image, therefore, does not show the azimuthal surface brightness variations observed at longer wavelengths. A similar result, albeit with a lower $\mathrm{S} / \mathrm{N}$, is obtained with the $160 \mu \mathrm{m}$ image (Figure 2(b)).

\subsubsection{MIPS SED-Mode Source Size}

Figure 3(a) shows the MIPS SED-mode source FWHM (along the slit) versus wavelength from Gaussian fits to the spatial profiles of the signal at each row of the mosaic image. The FWHM are $21,23.5$, and $38^{\prime \prime}$ at 55,70 , and $90 \mu \mathrm{m}$, respectively, consistent with the 70 and $160 \mu \mathrm{m}$ image source sizes. Analysis of spectrophotometric data from the MIPS-SED instrument, presented in Figure 3(b), is described in Sections 3.3.1 and 4.3.2. The small-scale variations in FWHM and in flux density versus wavelength relative to simple monotonic trends, as well as the steep increase in FWHM longward of $87 \mu \mathrm{m}$, are not real but represent systematic uncertainties in the reduction procedures, which dominate the photometric uncertainties.

\subsubsection{CSO Sub-mm Map}

Figure 4 displays the CSO SHARC II $350 \mu \mathrm{m}$ map. The sub-mm ring that has been previously observed at 450 and $850 \mu \mathrm{m}$ is apparent here, with approximately the same spatial scale. The $350 \mu \mathrm{m}$ image has flux consistent with zero excess in the central beam (FWHM $=29$ AU) after subtraction of a model $8 \mathrm{mJy}$ point source corresponding to the stellar photosphere emission, representing $2 \%$ of the total flux in the map (Table 1). The $350 \mu \mathrm{m}$ ring may show north-south extension, as in the Spitzer 70 and $160 \mu \mathrm{m}$ images and the James Clerk Maxwell Telescope (JCMT) $450 \mu \mathrm{m}$ map, but differing from the $850 \mu \mathrm{m}$ morphology.

The average value and dispersion of the surface brightness around the ring at $350 \mu \mathrm{m}$ is $S_{v}=0.15 \pm 0.04 \mathrm{mJy}$ per square arcsec. The brightest $350 \mu \mathrm{m}$ spot, in the north to north-east sector of the ring, has $S_{v} \sim 0.24 \mathrm{mJy}$ per square arcsec, about $2 \sigma$ above the average. The location and shape of the $350 \mu \mathrm{m}$ ring are confirmed by the 2003 and 2005 data sets if they are independently analyzed, but the azimuthal substructure is not.

\subsection{Broadband Photometry}

\subsubsection{Stellar Photosphere}

Precise determination of $\epsilon$ Eri's photospheric SED was necessary to measure the excess emission from the disk, especially in the mid-IR where the system SED is only slightly above the stellar continuum. The star has $m_{v}=+3.74, B-V=+0.88$ (Mendoza et al. 1978), and $V-K=+2.11$ (Koornneef 1983a). Average main-sequence $B-V$ colors are +0.82 and +0.92 for K0 and K2 temperature classes, respectively (Johnson 1986). Average $V-K$ colors are +2.00 and +2.25 for $\mathrm{K} 1$ and $\mathrm{K} 2$, respectively (Koornneef 1983b). These data imply that $\epsilon$ Eri is a little bluer than the average $\mathrm{K} 2 \mathrm{~V}$ star, with an interpolated temperature class between K1 and K2. Flower (1996) gave $T_{\text {eff }}=5090 \mathrm{~K}$ for $B-V=0.88$.

Flux densities from $\epsilon$ Eri at 3.6, 4.5, 5.7, and $7.9 \mu \mathrm{m}$ that are determined by PSF-fitting of Spitzer IRAC data (Section 2.1) have ratios nicely consistent with expectations for a photosphere of this star's temperature and luminosity. The photosphere model SED used in this paper is a Kurucz model for $T=5000 \mathrm{~K}$ (nearest match to $5090 \mathrm{~K}$ ) and $\log g=4.5$, normalized to the four IRAC flux densities via least-squares minimization. The rms of the fit to those points is $1.5 \%$. The result is consistent with ground-based photometry at $2.2 \mu \mathrm{m}$ and shorter wavelengths to better than $2 \%$ precision. Figure 5 displays the IRAC data points and fitted photosphere in relation to other broadband measurements discussed below. Table 1 presents extrapolated photospheric flux densities, which can be regarded as having $2 \%$ uncertainty.

\subsubsection{Photometry from Image Data}

Since the disk is not resolved at $24 \mu \mathrm{m}$, the total emission from the disk at that wavelength was obtained using both 

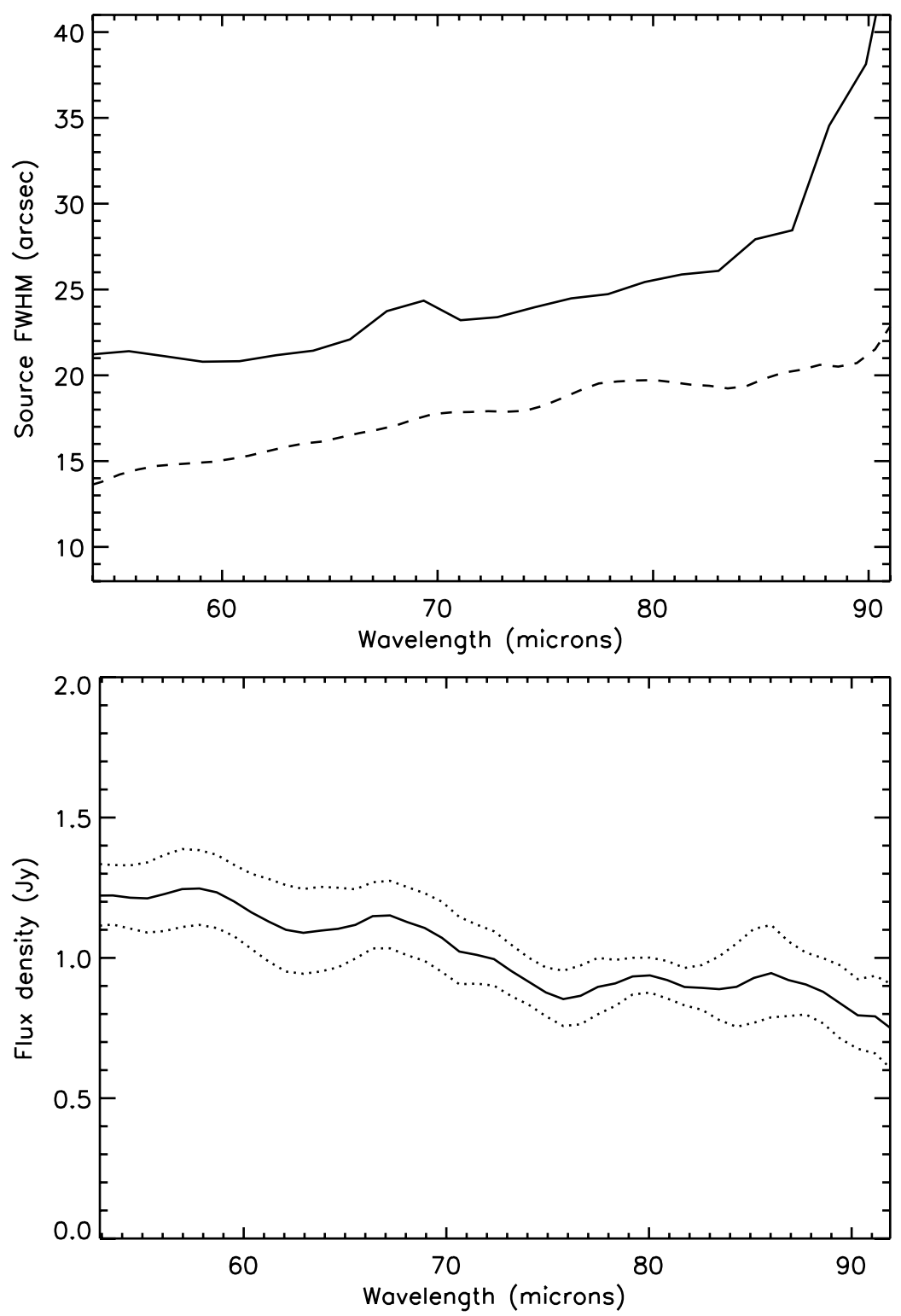

Figure 3. MIPS SED-mode measurements of $\epsilon$ Eri. (a) (solid line) FWHM of the $\epsilon$ Eri disk emission vs. wavelength, extracted by Gaussian fitting along the slit at P.A. $85^{\circ}$, vs. (dashed line) the same for Canopus, an unresolved calibration star with no IR excess. $\epsilon$ Eri is clearly resolved across the wavelength range of the MIPS SED measurements. (b) Star+disk spectrum windowed by the $20^{\prime \prime}$ wide slit, without aperture correction. The dotted lines indicate the range of photometric uncertainty based on uncertainty in Gaussian fits across the slit. Subsequent model-dependent flux normalization of these data is discussed in Section 4.4.2.

aperture photometry and PSF-fitting photometry optimized for point sources; for details, see Su et al. (2006). A total of $2.04 \pm 0.04$ Jy was estimated for the star and disk combined, which is $18 \%$ above the expected photosphere. No color correction was applied at $24 \mu \mathrm{m}$ because the observed SED slope in that wavelength region implied a MIPS filter correction factor of $\sim 1$.

The total flux density of the system at 70 and $160 \mu \mathrm{m}$ was integrated over a circular aperture with a radius of $64^{\prime \prime}$ centered at the star. The total disk emission in the $70 \mu \mathrm{m}$ and $160 \mu \mathrm{m}$ bands is 1.50 and $0.93 \mathrm{Jy}$, respectively, after application of color correction factors of 0.893 and 0.971 , appropriate for a $55 \mathrm{~K}$ blackbody.

IRAS photometry of $\epsilon$ Eri spans an important range of wavelengths, from the red end of the photospheric SED to beyond the peak of the excess. The Spitzer IRS spectrum, MIPS images, and MIPS SED-mode data allow a refined estimate of the continuum slope across the IRAS 25 and $60 \mu \mathrm{m}$ bands and, hence, more accurate color corrections, which were applied here.

Figure 5 and Columns (1) and (2) of Table 1 present the merged system (star+disk) broadband SED including Spitzer MIPS photometry from image data, integrated flux density from the CSO $350 \mu \mathrm{m}$ map, newly color-corrected IRAS catalog flux densities, ISO $200 \mu \mathrm{m}$ flux density, and previously published integrated flux densities at 450 and $850 \mu \mathrm{m}$ from Greaves et al. (2005). The peak of the SED is between $\lambda \sim 70$ and $100 \mu \mathrm{m}$.

ISO photometry of $\epsilon$ Eri (Walker \& Heinrichsen 2000) yielded a flux density of $1880 \mathrm{mJy}$ at $200 \mu \mathrm{m}$ (cf. the predicted photosphere value of $25 \mathrm{mJy}$ ), far above the $160 \mu \mathrm{m}$ point. The Spitzer $160 \mu \mathrm{m}$ image and the 450 and $850 \mu \mathrm{m}$ sub-mm maps show significant background emission extended beyond $40^{\prime \prime}$ from the star that would have been included in the large long-wavelength effective beam sizes of both ISO $(200 \mu \mathrm{m}$ photometric aperture diameter $\left.90^{\prime \prime}\right)$ and IRAS $(100 \mu \mathrm{m}$ detector footprint $\left.\sim 180^{\prime \prime} \times 300^{\prime \prime}\right)$. Numerous objects with NIR colors 
Table 1

$\epsilon$ Eri Broadband Photometry

\begin{tabular}{|c|c|c|c|c|}
\hline$\lambda(\mu \mathrm{m})$ & Total $F_{v}(\mathrm{Jy})$ & $\operatorname{Star} F_{v}(\mathrm{Jy})$ & Excess $F_{v}(\mathrm{Jy})$ & Notes \\
\hline 3.550 & $64.320 \pm 1.140$ & 64.320 & $\ldots$ & IRAC \\
\hline 4.493 & $39.800 \pm 0.700$ & 39.800 & $\ldots$ & IRAC \\
\hline 5.731 & $26.110 \pm 0.470$ & 26.110 & $\ldots$ & IRAC \\
\hline 7.872 & $14.910 \pm 0.260$ & 14.910 & $\ldots$ & IRAC \\
\hline 12.000 & $6.690 \pm 0.540$ & 6.570 & 0.130 & Color-corrected IRAS \\
\hline 23.680 & $2.042 \pm 0.040$ & 1.726 & 0.315 & MIPS \\
\hline 25.000 & $1.900 \pm 0.280$ & 1.550 & 0.350 & Color-corrected IRAS \\
\hline 60.000 & $1.750 \pm 0.250$ & 0.270 & 1.480 & Color-corrected IRAS \\
\hline 71.420 & $1.688 \pm 0.207$ & 0.189 & 1.499 & MIPS \\
\hline 100.000 & $1.990 \pm 0.300$ & 0.100 & 1.890 & Color-corrected IRAS \\
\hline 156.000 & $0.970 \pm 0.186$ & 0.039 & 0.931 & MIPS \\
\hline 200.000 & $1.880 \pm 0.280$ & 0.020 & 1.860 & $\mathrm{ISO}^{1}$ \\
\hline 350.000 & $0.366 \pm 0.050$ & 0.008 & 0.358 & CSO/SHARC II \\
\hline 450.000 & $0.250 \pm 0.020$ & 0.005 & 0.245 & JCMT/SCUBA $^{2}$ \\
\hline 850.000 & $0.037 \pm 0.003$ & 0.001 & 0.036 & $\mathrm{JCMT} / \mathrm{SCUBA}^{2}$ \\
\hline
\end{tabular}

References: (1) Walker \& Heinrichsen 2000; (2) Greaves et al. 2005.

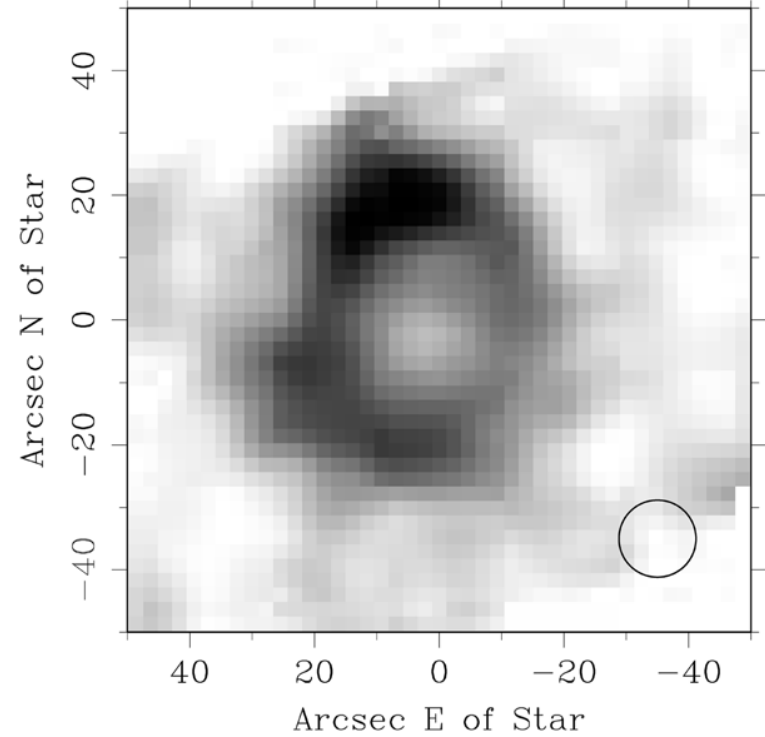

Figure 4. CSO SHARC II $350 \mu \mathrm{m}$ map. Dark is positive. The circle's diameter indicates 12 ". 7 resolution to which the map was smoothed, equivalent to $41 \mathrm{AU}$ at the distance of $\epsilon$ Eri. A scaled PSF representing the relatively small $350 \mu \mathrm{m}$ brightness of the central star has been subtracted.

of galaxies that would be strong sources at $\lambda \gtrsim 100 \mu \mathrm{m}$ are detected in Spitzer IRAC images of the $\epsilon$ Eri field (Marengo et al. 2006). The presence of this complex background emission probably accounts for the high flux density measured by ISO; the present observations indicate that much of the ISO flux must come from beyond the sub-mm ring. The IRAS $100 \mu \mathrm{m}$ and ISO $200 \mu \mathrm{m}$ photometry (Figure 5), therefore, provide upper limits to the system flux densities at those wavelengths. A less confused view of the source at 100-200 $\mu \mathrm{m}$ should be eventually possible with deep Herschel PACS and SOFIA HAWC observations.

\subsection{Spectrophotometric Data}

\subsubsection{MIPS SED-Mode}

With $\epsilon$ Eri expected to appear as an extended source along the MIPS SED slit, simple aperture photometry measurements cannot be made without admitting significant background noise.
To address this problem, a Gaussian was fit to the spatial profile of the signal along each row of the SED mosaic. The integrated product of the Gaussian width and height gives the source response at each wavelength. The resulting $\epsilon$ Eri spectrum in instrumental units was converted to physical units by reducing and extracting an archival spectrum of the calibration star Canopus ( $\alpha$ Car, G8 III) in the same way. The Canopus spectrum was normalized to give a flux density of $2.15 \mathrm{Jy}$ at $70 \mu \mathrm{m}$ (the star's $3.11 \mathrm{Jy}$ source flux density multiplied by a $69 \%$ slit throughput factor appropriate for a point source). The result was then corrected for the spectral response function by assuming that Canopus has a Rayleigh-Jeans spectral slope in the FIR. The flux normalization and spectral response function derived from Canopus were then applied to the $\epsilon$ Eri spectrophotometry.

Figure 3(b) presents the resulting MIPS SED-mode spectrum from 55 to $90 \mu \mathrm{m}$, giving the detected flux density transmitted through the MIPS SED slit independent of assumptions about the true spatial extent of the source. The slope of the spectrum, declining to longer wavelengths, is due to the intrinsic shape of the star+disk SED combined with wavelength-dependent slit losses as the size of the diffracted and extended emission region varies relative to the $20^{\prime \prime}$ wide MIPS SED entrance aperture. Linking the MIPS SED-mode spectrophotometric data to a disk model is described in Section 4.3.2.

$$
\text { 3.3.2. IRS }
$$

Spitzer IRS spectrophotometric data for the disk plus stellar photosphere are included in Figure 5. The photosphere model described in Section 3.2.1 is overplotted for comparison. A constant offset of $173 \mathrm{mJy}$ was subtracted from the IRS data, determined by making the average excess above the photosphere model from 5 to $12 \mu \mathrm{m}$ equal to zero. This is consistent with the observed lack of significant excess emission in broadband photometry at 5.7, 7.9, and $12 \mu \mathrm{m}$ (Table 1). That offset represents only $1 \%-3 \%$ of the total source flux in that wavelength range, well within the IRS calibration uncertainty. The resulting rescaled IRS mid-IR SED is in close agreement with the MIPS $24 \mu \mathrm{m}$ image photometry and with the lowerprecision IRAS $25 \mu \mathrm{m}$ broadband point.

The Figure 5 panel inset shows the spectrum from 10 to $30 \mu \mathrm{m}$ after subtraction of the photosphere. Significant departure of the 


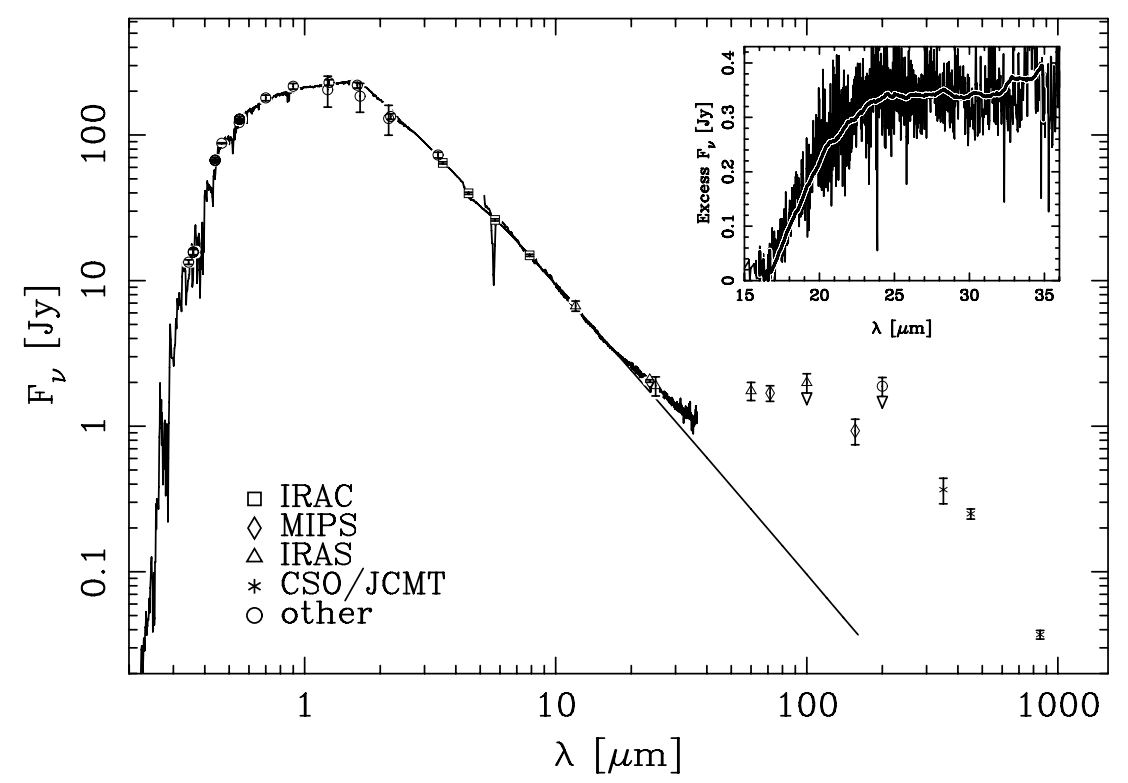

Figure 5. Merged broadband SED of the $\epsilon$ Eri system. Numerical values are presented in Table 1. The thin solid curve is a Kurucz photosphere for $T=5000 \mathrm{~K}$, $\log g=4.5$, fit to the four Spitzer IRAC photometric points at 3.6-7.9 $\mu \mathrm{m}$. The thick curve is the combined Spitzer IRS SL + SH + LH spectrum rescaled to have zero average excess at 5-12 $\mu \mathrm{m}$. The IRAS $100 \mu \mathrm{m}$ point and the ISO $200 \mu \mathrm{m}$ point are considered upper limits due to background contamination. The inset panel shows the mid-IR segment of the SED from Spitzer IRS (thin solid line), after subtraction of the photosphere model displayed in the main figure. The thick line is the same spectrum smoothed with a 111 point $(\sim 3.3 \mu \mathrm{m})$ boxcar.

$\epsilon$ Eri SED from the photosphere begins at about $15 \mu \mathrm{m}$. The mid-IR SED is remarkable in having a very steep rise from 15 to $20 \mu \mathrm{m}$, a "plateau" of almost constant flux density from about $20-30 \mu \mathrm{m}$, and then the beginning of another steep rise beyond $30 \mu \mathrm{m}$.

\section{MODELING}

\subsection{General Considerations}

The data presented above provide new information on the $\epsilon$ Eri circumstellar dust spatial distribution, new spectral information regarding warm dust components, and separation of disk emission from confusing background sources. A model of particle spatial distributions and physical properties can now be produced that will allow improved understanding of dust lifetimes and dynamics, which in turn allows inferences about the locations of planetary companions and about the evolution of this system for comparison with our solar system.

The modeling process involved (1) derivation of intrinsic 70,160 , and $350 \mu \mathrm{m}$ one-dimensional radial surface brightness profiles that, when convolved with the respective PSFs, match the observed radial profiles; (2) calculation of a physical model for the outer disk with specific grain spatial distributions, size distributions, and compositions that yields the intrinsic 70, 160, and $350 \mu \mathrm{m}$ surface brightnesses inferred from the first step; (3) derivation of another physical model for warm material detected by IRAS and Spitzer IRS but not resolved in the imaging data, and then testing that the predicted long-wavelength emission from the inner material would be consistent with the 70, 160 and $350 \mu \mathrm{m}$ radial profiles; and finally (4) checking that the overall physical model, including both resolved and unresolved material, correctly predicts the observed SED of IR excess from 15 to $850 \mu \mathrm{m}$ and also the MIPS SED-mode narrow-slit flux densities plus one-dimensional source sizes or limits. As mentioned in Section 3.3.1, the MIPS SED fluxes need to be corrected for slit losses, and that can only be done by iteratively estimating the corrections using synthetic images derived from the model (Section 4.3.2).
The $350 \mu \mathrm{m}$ map shows a deficit of sub-mm emission at $r \lesssim 35$ AU relative to 35-90 AU, similar to previous sub$\mathrm{mm}$ maps. In contrast, the 24,70 , and $160 \mu \mathrm{m}$ image profiles and photometry plus mid-IR spectrophotometry and broadband photometry, as well as the original IRAS detection, together indicate the presence of significant amounts of warm material lying at $r \lesssim 35 \mathrm{AU}$, within the central void in the sub-mm ring.

The surface brightness in the high-S/N $70 \mu \mathrm{m}$ images is continuous and monotonic out to $r \gtrsim 45 \operatorname{arcsec}(140 \mathrm{AU})$, far outside the sub-mm ring. However, trial PSF convolutions of model intrinsic brightness distributions revealed that the outer boundary of the $70 \mu \mathrm{m}$ emission is actually located at $r \sim 34^{\prime \prime}(110 \mathrm{AU})$, with apparently further extended emission representing the PSF wings. In comparison, nearly all of the observed sub-mm surface brightness is contained in the ring between about 35 and 90 AU (11-28"; Greaves et al. 2005; also see Schütz et al. 2004 regarding the source diameter at $1.2 \mathrm{~mm}$ ). The region outside the sub-mm ring, beyond $r=28^{\prime \prime}$ (90 AU), is here designated the "halo."

An outer limit to the $160 \mu \mathrm{m}$ emission is more difficult to determine due to background source confusion discussed above, but the data and preliminary modeling indicate that the overall spatial distribution of material observed at $160 \mu \mathrm{m}$ much more closely resembles the $70 \mu \mathrm{m}$ than the $350 \mu \mathrm{m}$ emission. Significant emission at $350 \mu \mathrm{m}$ does not extend beyond $28^{\prime \prime}(90 \mathrm{AU})$.

\subsection{Outer Fully-Resolved Disk}

\subsubsection{Radial Surface Brightness Profiles}

The $70 \mu \mathrm{m}$ images have small ellipticity and are also azimuthally smooth (Figure 2), justifying the use of onedimensional average radial profiles centered on the star position for detailed comparison of model surface brightness distributions with observations. Based on inspection of the radial profiles and preliminary modeling, fiducial radii of $r=11,28$, and $34^{\prime \prime}(35,90$, and $110 \mathrm{AU})$, representing transition points in some 

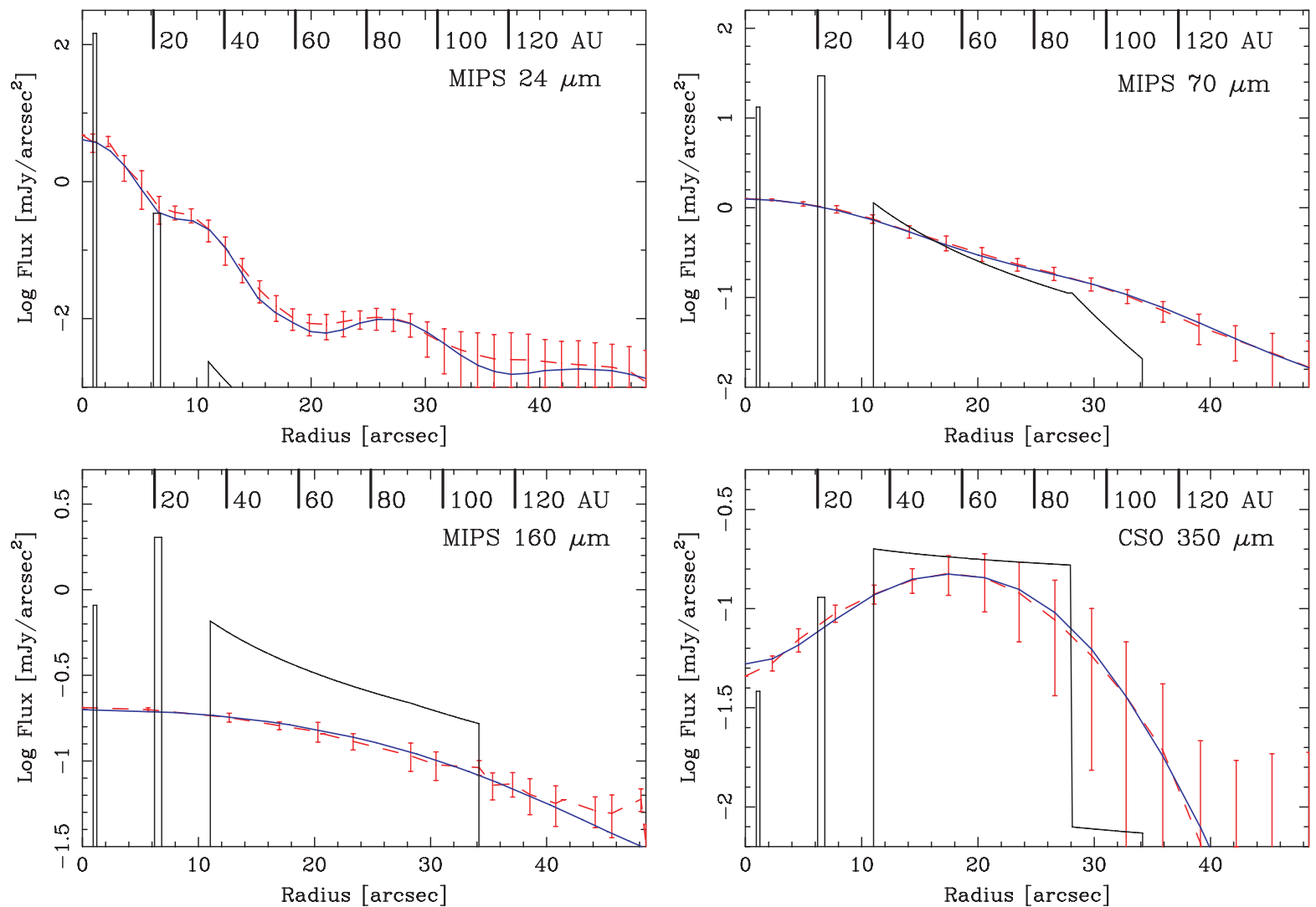

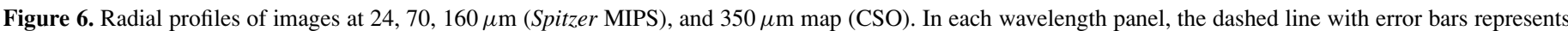

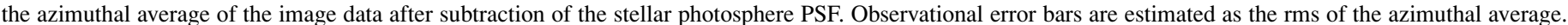

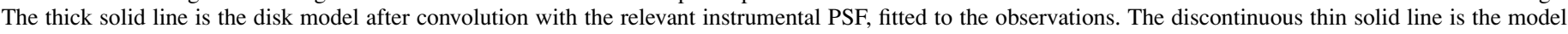
intrinsic surface brightness before PSF convolution (Section 4.2).

(A color version of this figure is available in the online journal.)

of the surface brightness distributions, were chosen as primary points for matching models to observations.

Intrinsic surface brightness profiles at 70,160 , and $350 \mu \mathrm{m}$ were constructed by choosing surface brightness values at the three fiducial radii and interpolating between those points with simple power laws. Production of model PSF-convolved radial profiles for trial-and-error comparison with the observations involved expansion of the model one-dimensional radial profiles into two-dimensional pseudo-images, convolution of each pseudo-image with the respective wavelength's twodimensional PSF derived for a point source with $\epsilon$ Eri's temperature using STinyTim (Krist 2006), and then collapsing the convolved model two-dimensional map back into a onedimensional profile. After each trial, differences between predicted and observed surface brightness profiles were used to estimate new values at the fiducial radii for the next trial.

The results of modeling the $24,70,160$, and $350 \mu \mathrm{m}$ surface brightness profiles are displayed in Figure 6. The actual locations of changes in profile slopes near the fiducial radii are uncertain to probably $10 \%$ in radius, that is, the modeling process had approximately that much spatial resolution. Note that the purpose of these intrinsic surface brightness profile determinations was merely to match the observed profiles, without reference at this step in the modeling process to physicallymotivated grain properties, which are treated in Section 4.2.2.

\subsubsection{Physical Grain Models}

The Heidelberg DDS code (Wolf \& Hillenbrand 2005) was used to estimate grain properties and surface densities that would yield the surface brightness values determined in the preceding modeling step. Grains were assumed to be solid (nonporous) and spherical. DDS settings represented $\epsilon$ Eri as a $5090 \mathrm{~K}$ blackbody with $0.33 L_{\odot}$. Choice of grain composition, grain minimum and maximum sizes, size-distribution power-law exponent, and mass surface densities at the three fiducial radii allowed calculation of emitted surface brightness for comparison with the intrinsic 70 , 160 , and $350 \mu \mathrm{m}$ surface brightness values determined at those locations. Integrated disk flux densities and grain masses could then be calculated using surface densities and grain properties interpolated between the values at the fiducial radii.

The large difference in appearance of the sub-mm maps versus the Spitzer 70 and $160 \mu \mathrm{m}$ images led to a search for model disk components such that (1) material having high sub-mm and low FIR emissivity would dominate in the sub-mm ring region, coexisting with (2) material having low sub-mm and high FIR emissivity extending across the ring into the halo region. Many combinations of grain properties, size distributions, and spatial distributions can match these constraints. A simple model can be built of components that separately fit the 70 and $160 \mu \mathrm{m}$ images and the $350 \mu \mathrm{m}$ map.

The final model for the resolved disk combines (1) large (radii $a=100-200 \mu \mathrm{m})$ amorphous $\mathrm{H}_{2} \mathrm{O}$ ice grains located in the sub$\mathrm{mm}$ ring, plus (2) small ( $a=6-23 \mu \mathrm{m})$ "astronomical" silicate grains having a broader spatial distribution that includes the ring. Experiments varying the value of the grain-size distribution exponent indicated that the conventional collision equilibrium value of $x=-3.5$ could be used for both populations although other values are not excluded. Net emissivities integrated over 
the size distributions are approximately equivalent to single-size particles with radii of $135 \mu \mathrm{m}$ and $15 \mu \mathrm{m}$ for the large icy and small silicate grains, respectively.

\subsection{Two Inner Warm Belts}

\subsubsection{Dust Properties and Locations}

Spitzer IRS, MIPS, and MIPS-SED data reveal the presence of material within the sub-mm ring's central void. PSF analysis of 70 and $160 \mu \mathrm{m}$ image surface brightness profiles shows emission closer to the star than $11^{\prime \prime}$ (35 AU) but also reveals that the emission does not extend all the way to $r=0$. The local maximum and plateau in the IR excess around 20 $30 \mu \mathrm{m}$ indicate emission at $T \sim 100-150 \mathrm{~K}$, confirming the location of material at $r<35 \mathrm{AU}$, because at $35 \mathrm{AU}, T_{\text {dust }}$ would be only $\sim 40 \mathrm{~K}$ for blackbody grains or $\sim 60 \mathrm{~K}$ for $1 \mu \mathrm{m}$ silicate grains. Quadrature subtraction of a point source size from the MIPS-SED one-dimensional source extent at $55 \mu \mathrm{m}$ (Figure 3(a)) yields an emission radius of very roughly $8^{\prime \prime}(25 \mathrm{AU})$.

Modeling the properties of the inner material involved first noting that the marked change in the SED slope at about $20 \mu \mathrm{m}$ (Figure 5) is easily explained as a silicate mineralogical band (e.g., Chen et al. 2006). Trial-and-error experiments with the Heidelberg DDS code showed that the detectability of the band sets a rough upper limit on grain radius $a \leqslant 20 / 2 \pi \sim 3 \mu \mathrm{m}$. In contrast, "generic" grains of that size without mineralogical attributes produce an emission SED that is significantly broader than observed, as do ice grains.

The 15-30 $\mu \mathrm{m}$ segment of the $\epsilon$ Eri SED can be approximately matched by emission from a belt of warm "astronomical" silicate grains with characteristic size $\sim 3 \mu \mathrm{m}$, located about $3 \mathrm{AU}\left(\sim 1^{\prime \prime}\right)$ from the star. This innermost warm belt is responsible for $\gtrsim 95 \%$ of the system's excess emission at $25 \mu \mathrm{m}$, first detected by IRAS. Note that dust located 3 AU from $\epsilon$ Eri would be entirely contained within the IRS slits that have a minimum width of 4 ".7 (15 AU).

The precise position of the " 3 AU" belt is constrained by relations among grain size, radiative efficiency, and temperature. For example, if the model grain size $a$ is set at $2.2 \mu \mathrm{m}$ instead of $3 \mu \mathrm{m}$, the belt must move to $r \sim 3.8$ AU to have the correct color temperature. However, grain efficiency declining steeply to longer wavelengths for such small grains would unacceptably "erode" the 20-30 $\mu \mathrm{m}$ SED plateau. Thus, the grain size and belt location are determined to perhaps $25 \%$ precision. There is no separate information regarding an upper limit to the belt's width, but that can be taken to be equivalent to the uncertainty in the belt location, that is, $\sim 25 \%$.

The rising IRS spectrum near $35 \mu \mathrm{m}$ (Figure 5) cannot be fit by emission from the material inferred at $\sim 3 \mathrm{AU}$, which has an SED decreasing beyond $30 \mu \mathrm{m}$. The steep upward slope from 35 to $60 \mu \mathrm{m}$, characterized by a color temperature of about $55 \mathrm{~K}$, plus the relatively narrow SED peak at 70 $100 \mu \mathrm{m}$, locates the next lower-temperature population of grains also in a belt with narrow ranges of temperature and particle size. The precise properties of this material are especially constrained by the MIPS SED-mode 55-90 $\mu \mathrm{m}$ flux densities, but because of slit losses, those data must be renormalized using models of the source geometry, as described in the next section.

A belt of $a \sim 8 \mu \mathrm{m}$ "astronomical" silicate grains located at $r \sim 20 \mathrm{AU}\left(\sim 6^{\prime \prime}\right)$ satisfies the SED and source size constraints. Models placing the belt at $20 \mathrm{AU}$ can be distinguished from alternate locations at 10 or $30 \mathrm{AU}$. Material at $20 \mathrm{AU}$ could also explain the slight filling-in of the first Airy dark ring at $24 \mu \mathrm{m}, r \sim 23 \mathrm{AU}$ (Figure 6(a)). Silicate grains of this size and temperature would not show mineralogical features in the Spitzer data, so the composition of this belt is consistent with, but not determined to be, silicate.

Models with continuous distributions of material extending across the sub-mm void produced too much total mid-IR and FIR flux and/or did not match the surface brightness profiles and source size measurements. The warm material is clearly restricted to annular zones with relatively empty gaps.

\subsubsection{Modeling Details, Including MIPS SED Normalization}

Convergence on final models for the two inner warm belts involved iterative comparison of model SEDs from the Heidelberg DDS software with the IRS 15-35 $\mu \mathrm{m}$ and 55-90 $\mu \mathrm{m}$ (MIPS SED-mode) flux densities that are not already accounted for by the model of the resolved disk. Trial models yielded intrinsic surface brightness profiles at the observed wavelengths that were (1) convolved with the $24 \mu \mathrm{m}$ PSF to compare with the observed unresolved source, (2) convolved with a derived Spitzer $55 \mu \mathrm{m}$ PSF and MIPS SED-mode slit mask to compare with the observed source $55 \mu \mathrm{m}$ one-dimensional FWHM, and (3) added to the already-established 70,160 , and $350 \mu \mathrm{m}$ intrinsic surface brightness profiles of the disk and then PSF-convolved to check that the profiles were not significantly perturbed by the extra warm material.

The model properties of these two belts within the sub-mm void interact, in that they both produce significant flux density at $\lambda=30-35 \mu \mathrm{m}$. For example, changing the second warm belt's properties toward smaller spatial radius and/or particle radii produces greater $30-35 \mu \mathrm{m}$ model emission, requiring the innermost belt to have greater spatial radius and/or particle radii so as to preserve the total mid-IR SED shape.

The slit losses and flux normalization of the MIPS SED data were derived by generating two-dimensional synthetic images of the source using model radial profiles of the disk computed at the MIPS SED wavelengths. This analysis was restricted to the two extremes of the MIPS SED wavelength range, 55 and $90 \mu \mathrm{m}$, using the midpoint at $70 \mu \mathrm{m}$ as the flux normalization reference.

Synthetic two-dimensional images at 55, 70, and $90 \mu \mathrm{m}$ were derived by convolving azimuthal projections of the radial disk model profiles with the STinyTIM PSF, and then adding a point source rescaled to the stellar flux at each wavelength. The resulting $70 \mu \mathrm{m}$ model "image" closely matches the $70 \mu \mathrm{m}$ observations (Figure 6(b)). A software mask with the size of the MIPS SED slit was then centered on the synthetic images to measure the fraction $q_{v}$ of the total flux recovered through the SED-mode slit: $58 \%, 47 \%$, and $38 \%$, at 55, 70, and $90 \mu \mathrm{m}$, respectively. The overall flux normalization factor $N_{70}^{\text {flux }}$ at $70 \mu \mathrm{m}$ is derived by rescaling the SED in Figure 3(b) to have a $70 \mu \mathrm{m}$ flux density identical to the MIPS $70 \mu \mathrm{m}$ image total flux density (1.688 Jy; Table 1$)$, which yields $N_{70}^{\text {flux }} \simeq 1.78$. The correction for the other two wavelengths can be derived by factoring out the slit loss at $70 \mu \mathrm{m}$ and multiplying by the slit losses at 55 or $90 \mu \mathrm{m}$ :

$$
N_{v}^{\text {flux }}=N_{70}^{\text {flux }} \times \frac{q_{v}}{q_{70}} .
$$

This procedure produced corrected MIPS SED flux densities of $1.62 \pm 0.25 \mathrm{Jy}$ at $55 \mu \mathrm{m}$ and $1.64 \pm 0.25 \mathrm{Jy}$ at $90 \mu \mathrm{m}$. Uncertainties were estimated by quadrature addition 


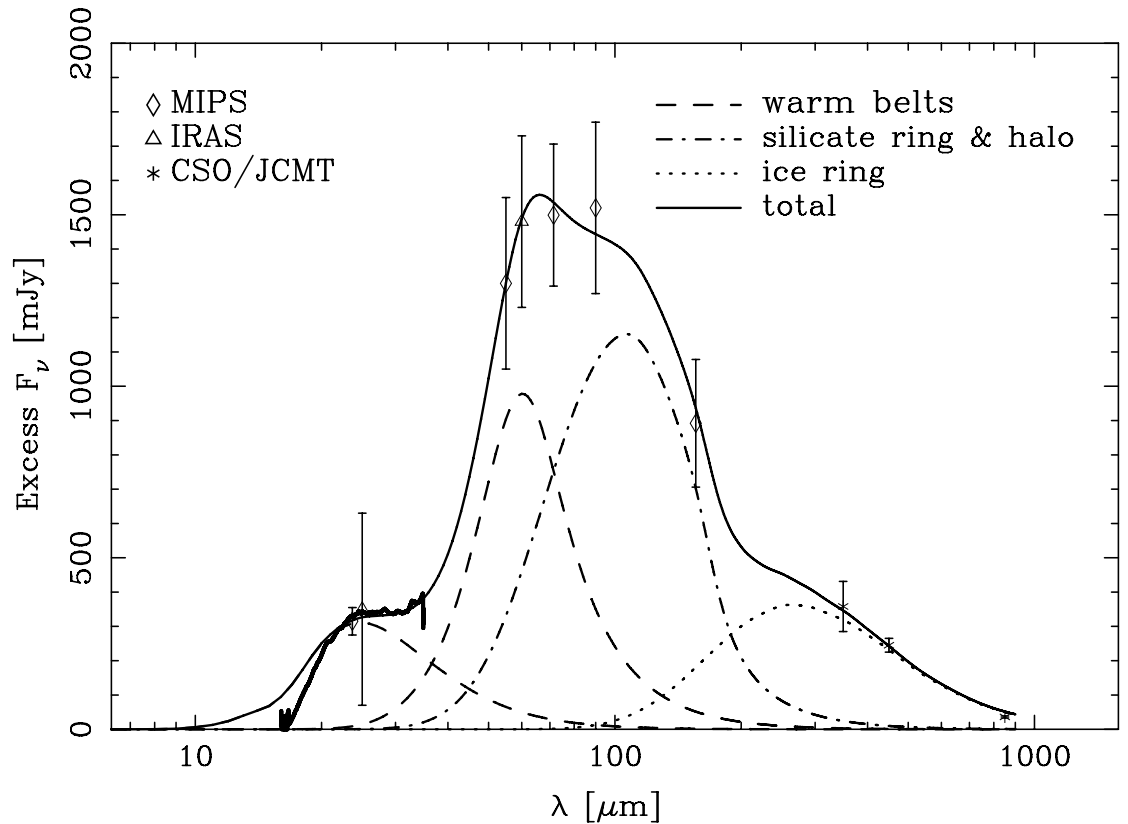

Figure 7. Observed SED of the $\epsilon$ Eri disk, after subtraction of the stellar photosphere, compared with the model SED. Individual photometric points include MIPS SED flux densities at 55 and $90 \mu \mathrm{m}$, iteratively aperture-corrected using model images, and rescaled according to the measured MIPS $70 \mu \mathrm{m}$ image total flux. The thick solid line is the IRS SL + SH + LH combined spectrum, rescaled to have zero average excess for $\lambda=5-12 \mu$ m. The thin solid line is the total model flux (sum of all four dust components). The two dashed lines are the contributions of the two unresolved inner belts. The dot-dashed line is the contribution of the small $(a \sim 15 \mu \mathrm{m})$ silicate grains in the sub-mm ring and halo. The dotted line is the contribution of the large $(a \sim 135 \mu \mathrm{m})$ ice grains in the sub-mm ring.

Table 2

Model Components

\begin{tabular}{lcccccc}
\hline \hline Component & $r(\mathrm{AU})$ & $M_{\mathrm{T}}\left(M_{\oplus}\right)$ & $\alpha$ & $a(\mu \mathrm{m})$ & $x$ & $f$ \\
\hline W1 & 3 & $1.8 \times 10^{-7}$ & $\ldots$ & 3.0 & $\ldots$ & $3.3 \times 10^{-5}$ \\
W2 & 20 & $2.0 \times 10^{-5}$ & $\ldots$ & 8.0 & $\ldots$ & $3.4 \times 10^{-5}$ \\
RS & $35-90$ & $2.0 \times 10^{-4}$ & +0.01 & $6.0-23$ & -3.5 & $3.0 \times 10^{-5}$ \\
RL & $35-90$ & $4.2 \times 10^{-3}$ & +1.05 & $100-200$ & -3.5 & $4.4 \times 10^{-6}$ \\
HS & $90-110$ & $2.5 \times 10^{-4}$ & +0.15 & $15-23$ & -3.5 & $4.8 \times 10^{-6}$
\end{tabular}

Notes. Columns: (1) model component: $\mathrm{W} 1=$ warm belt $1, \mathrm{~W} 2=$ warm belt $2, \mathrm{RS}=$ sub-mm ring, small grains; RL = sub-mm ring, large grains; $\mathrm{HS}=$ halo, small grains; (2) location; (3) total mass; (4) mass surface density exponent, assumed to be zero for the W1 and W2 components, fitted to data for the other components; (5) grain radius; (6) assumed grain size distribution exponent; (7) fractional luminosity, $L_{d} / L_{*}$.

of the $70 \mu \mathrm{m}$ uncertainty to the uncertainties in the uncorrected flux densities plotted in Figure 3(b). Once the photospheric contribution is subtracted, an excess from the disk of $1.30 \pm 0.25 \mathrm{Jy}$ at $55 \mu \mathrm{m}$ and $1.52 \pm 0.25 \mathrm{Jy}$ at $90 \mu \mathrm{m}$ is obtained.

\subsection{Model Summary}

As described in the preceding section, the overall model of $\epsilon$ Eri's circumstellar material includes (1) particles with low FIR emissivity and high sub-mm emissivity, consistent with the properties of radius $a=100-200 \mu \mathrm{m}$ (effective $a \sim 135 \mu \mathrm{m})$ amorphous $\mathrm{H}_{2} \mathrm{O}$ ice grains in the sub-mm ring at $r=35-90 \mathrm{AU}$; (2) particles with high FIR emissivity and low sub-mm emissivity, consistent with the properties of $a=$ 6-23 $\mu \mathrm{m}$ (effective $a \sim 15 \mu \mathrm{m}$ ) "astronomical" silicate grains at $r=35-110 \mathrm{AU}$, corresponding to the sub-mm ring plus an exterior halo; (3) a narrow belt at $\sim 3 \mathrm{AU}(T \sim 120 \mathrm{~K})$ of small $(a \sim 3 \mu \mathrm{m})$ silicate grains; and (4) a narrow belt at $\sim 20 \mathrm{AU}$ $(T \sim 55 \mathrm{~K})$ of small grains $(a \sim 8 \mu \mathrm{m})$ of undetermined, but possibly silicate, composition. Specific properties of the model components are presented in Table 2.

Figure 7 displays the SED of the complete model and the separate disk components compared with the photometric and spectrophotometric data (model photosphere SED subtracted). Spitzer mid-IR and FIR data especially reveal a complicated SED shape that strongly constrains the temperatures, locations, and grain sizes of warm unresolved material. The model is not unique but was built from the fewest components with the simplest assumptions that produced a good match to all the available data. Many alternate models were tested, resulting in confidence that (1) 70 and $160 \mu \mathrm{m}$ emission does not extend beyond $110 \mathrm{AU}$, (2) $350 \mu \mathrm{m}$ emission does not extend beyond $90 \mathrm{AU}$, (3) $350 \mu \mathrm{m}$ emission does not extend inside $35 \mathrm{AU}$, (4) the dominant emission in the sub-mm ring is not from silicate particles, (5) 70 and $160 \mu \mathrm{m}$ emission within $35 \mathrm{AU}$ must include nonemitting gaps, with emission restricted to annular zones, and (6) grains in the innermost warm belt at $r \sim 3 \mathrm{AU}$ have silicate composition.

The model rises less steeply than the IRS observations at $10-18 \mu \mathrm{m}$. The excess flux in the model is less than $3 \%$ of the emission from the system in that range. This may be related to uncertainty in the photospheric model subtraction at short wavelengths where the IR excess is barely significant, and may also indicate the presence of crystalline silicates, with a sharp $20 \mu \mathrm{m}$ spectral feature, combined with the amorphous silicates assumed in the model. 

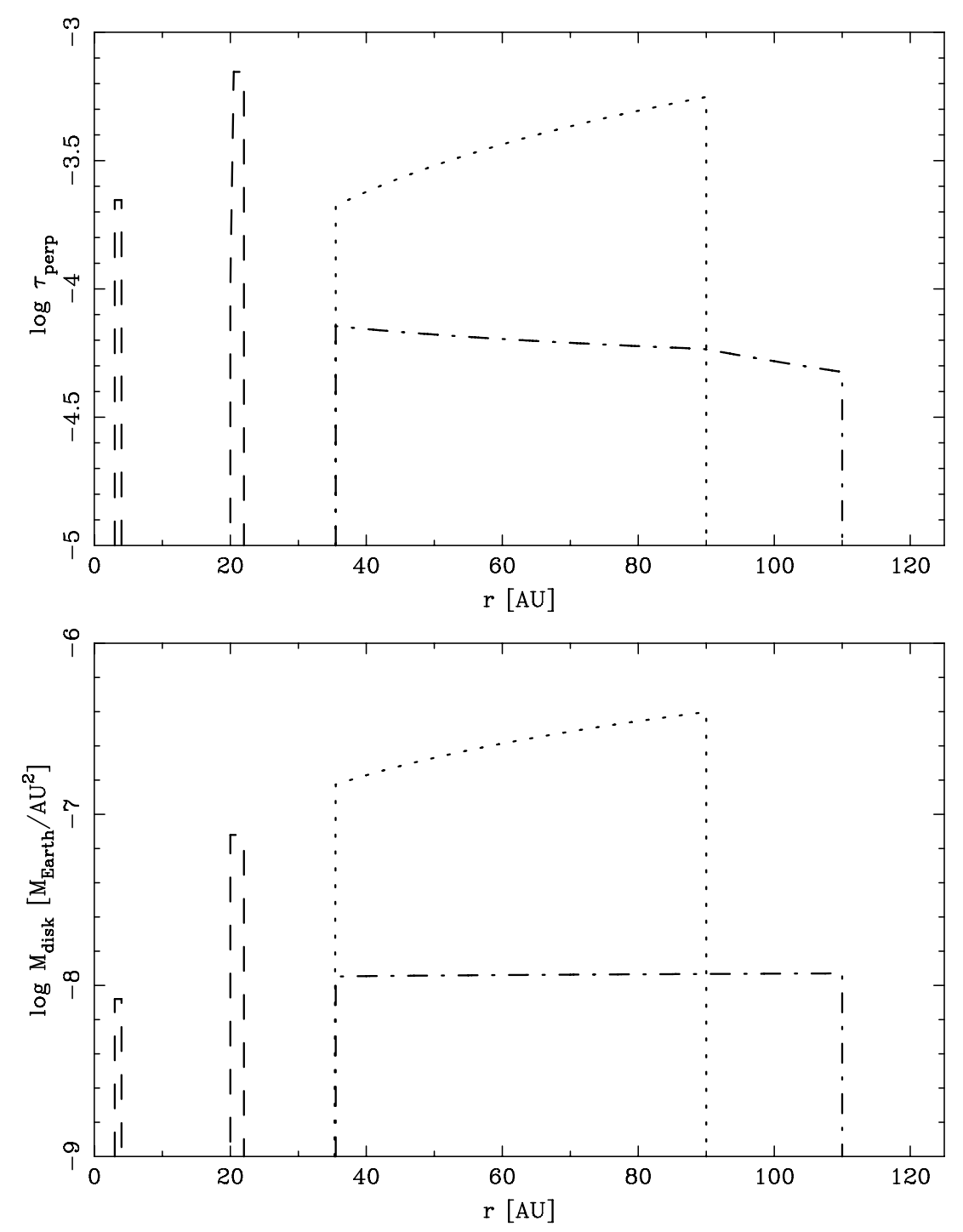

Figure 8. (a) Perpendicular optical depth $\tau_{\perp}$ vs. position for the five model disk components. The two unresolved inner belts (dashed lines), outer "icy" ring (dotted line), and ring+halo "silicate" dust populations (dot-dashed line) are shown. (b) Mass surface density vs. radius corresponding to the optical depth profiles in panel (a), assuming grain compositions and material densities described in the text. The difference in slopes between the optical depth and mass surface density profiles is due to radial variation in model particle properties.

The top panel of Figure 8 displays the model radial profile of perpendicular optical depth $\tau_{\perp}$, that is, fractional (unitless) surface density $\left(\mathrm{m}^{2}\right.$ of grain cross-sectional area per $\mathrm{m}^{2}$ of disk surface area; e.g., Backman 2004), and in the bottom panel, the corresponding mass surface density inferred from the optical depth and model grain properties is displayed. The slopes of the two functions across the sub-mm ring and halo are not equal because the model includes smooth variation in the particle size distribution between the fiducial radii, so the ratio of particle cross section (and thus optical depth) to mass surface density varies slightly with radius. The optical depths and mass densities of the two inner rings in Figure 8 are calculated by assuming $\Delta r$ values of $1 \mathrm{AU}$ at $3 \mathrm{AU}$ and $2 \mathrm{AU}$ at $20 \mathrm{AU}$; they would scale inversely with $\Delta r$.

The total inferred mass in detected grains, that is, with sizes from a few microns to a few hundred microns, carrying most of the radiating surface area, is roughly $5 \times 10^{-3} M_{\oplus} \sim 0.4 M_{\text {Luna }}$, $90 \%$ of which is in the large ("icy") grain component of the sub$\mathrm{mm}$ ring. The total bolometric fractional luminosity $\left(L_{d} / L_{*}\right)$ of all the disk components is $1.1 \times 10^{-4}$.
Figure 9 is a two-dimensional representation of the $\epsilon$ Eri debris disk system with a linear spatial scale, showing the sub-mm ring, halo, and inner warm belts. The position of the innermost warm belt is compared in the inset figure with one orbit solution for the candidate radial velocity planet (Section 5.4).

\section{DISCUSSION}

\subsection{Some Implications of the Disk Model}

The derived surface-density profile for the small grain component (Figure 8) has a nearly constant value from 35 to $110 \mathrm{AU}$, consistent with dynamics controlled by $\mathrm{P}-\mathrm{R}$ radiation drag or its corpuscular wind analog (Section 5.3). In contrast, the large-grain component has surface density rising from 35 to $90 \mathrm{AU}$. The larger grains are less subject to drag forces, so their radial distribution may reflect that of the underlying parent-body population.

The void interior to the sub-mm ring is inferred to be partly filled by two additional belts of particles with mean sizes 


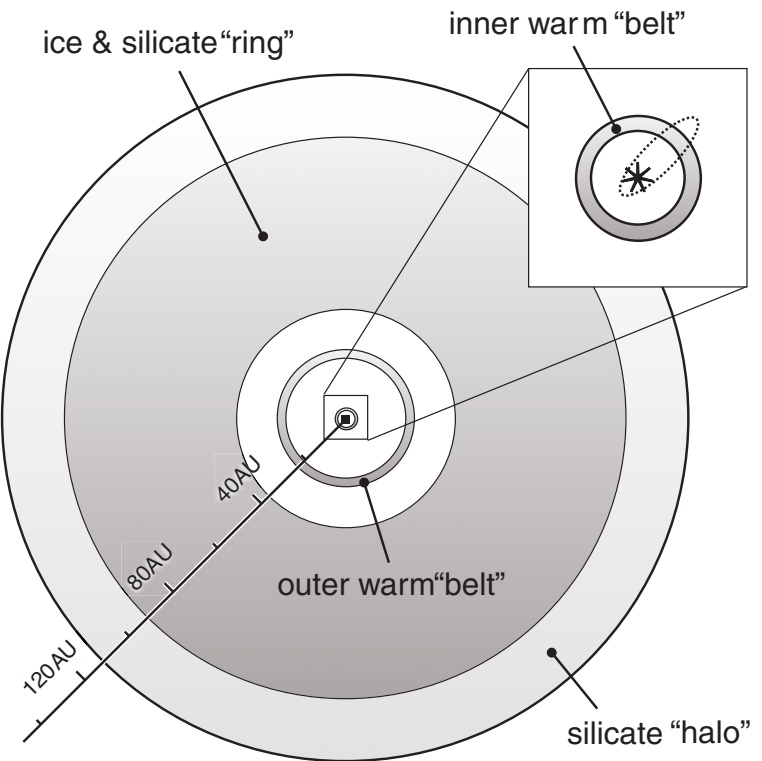

Figure 9. Representation of the $\epsilon$ Eri debris disk model components. The smallscale dotted ellipse is one solution for the orbit of the suggested radial velocity planet that appears to be inconsistent with the innermost warm debris belt's position.

smaller than in the sub-mm ring in both cases. The large grain population in the ring appears to be absent in the void, even though $100 \mu \mathrm{m}$ ice grains could persist against sublimation for as long as $\epsilon$ Eri's age, $850 \mathrm{Myr}$, at distances as small as $3 \mathrm{AU}$ from the star (see Equations 16 and 17 in Backman \& Paresce 1993 for expressions of ice sublimation timescales). Moreover, the mid-IR SED, plus the $70 \mu \mathrm{m}$ surface brightness profile in particular, indicates that there are gaps in the distribution of material from just beyond $3 \mathrm{AU}$ to almost $20 \mathrm{AU}$, and from just beyond $20 \mathrm{AU}$ to $35 \mathrm{AU}$. A mechanism is needed to prevent significant amounts of both small and large grains from drifting via $\mathrm{P}-\mathrm{R}$ drag from the sub-mm ring into the inner void (Section 5.4).

The total mass of detected grains in $\epsilon$ Eri's innermost belt is about $0.1 \times$ the mass estimated for the warm belt detected around the 230 Myr-old A star, $\zeta$ Lep (Chen \& Jura 2001). The size and structure of the $\epsilon$ Eri system, with two warm belts in a central void surrounded by a cold disk, and silicate dust identified in the innermost ring, bear striking resemblance to the circumstellar material in the much younger HD 113766 system (10-16 Myr, F3/F5 binary; Lisse et al. 2008).

An upper limit to the ratio of ice versus silicate grain mass for the sub-mm ring region in which they co-exist is about 16 . This ratio is intermediate between values for solar composition ( 50) and for comets ( 2-5; e.g., Altenhoff et al. 2002).

\subsection{Comparison with Prior Studies of $\epsilon$ Eri}

\subsubsection{Sub-mm Ring Features}

As noted above, the sub-mm ring at $350 \mu \mathrm{m}$ (Figure 4) has the same inner and outer diameters observed in previously published 850 and $450 \mu \mathrm{m}$ maps (Greaves et al. 1998; 2005 ), but $350 \mu \mathrm{m}$ ring surface brightness features do not, in general, align with 450 or $850 \mu \mathrm{m}$ features. The (JCMT/ Submillimeter Common-User Bolometric Array, SCUBA) 450 and $850 \mu \mathrm{m}$ data were obtained via dithered ("jiggle") mapping, whereas the (CSO/SHARC II) $350 \mu \mathrm{m}$ map was made via continuous spiral scanning. Real features should be robust against such differences in data acquisition and reduction procedures.

The strongest 450 and $850 \mu \mathrm{m}$ feature, about $1.5 \times$ brighter than the ring average, is in the south-east sector. A south-east feature appeared in some form in all the maps and was suggested to be a real part of the $\epsilon$ Eri system by Greaves et al. (2005) based on its motion over the 1997-2002 time span. Poulton et al. (2006) made a careful statistical analysis of this issue. Only a weak feature is seen at that location in the $350 \mu \mathrm{m}$ image. Other fairly prominent $850 \mu \mathrm{m}$ ring features, especially in the south-west sector, were considered by Greaves et al. (2005) to be background sources although they have no clear counterparts at $450 \mu \mathrm{m}$.

The strongest $350 \mu \mathrm{m}$ feature is located in the northnortheast section of the ring, with a surface brightness of about $0.24 \mathrm{mJy}$ per square arcsec. Its location approximately corresponds to a weak $450 \mu \mathrm{m}$ feature and a moderately strong $850 \mu \mathrm{m}$ feature that could also be part of the $\epsilon$ Eri system, considering proper motion plus orbital motion. Recall (Section 3.1.3) that the $350 \mu \mathrm{m}$ data, when split into equal parts and composed into separate maps, have about the same level of disagreement regarding azimuthal features as between maps at different wavelengths, and between the $850 \mu \mathrm{m}$ maps for 19972002 versus 2000-2002 (Greaves et al. 2005). As discussed in Section 3.1.3 and displayed in Figure 2, no surface brightness features are seen in the ring at 70 and $160 \mu \mathrm{m}$.

Real density features need not have the same surface brightness ratios versus wavelength as the main ring. For example, smaller grains trapped in planetary resonances can have increased libration widths, smoother distributions, and show less conspicuous clumping than large grains, so that some features prominent at long wavelengths may be less apparent at short wavelengths (Wyatt 2006). Azimuthal features with a surface brightness that does not vary progressively with wavelength, however, are difficult to reconcile with physical models.

The disagreement between details of the different sub-mm maps illustrates the difficulty of working at wavelengths with large variable sky opacity and emission. These comparisons imply that the Spitzer FIR images and CSO $350 \mu \mathrm{m}$ maps cannot confirm the ring surface brightness features reported at 450 or $850 \mu \mathrm{m}$.

\subsubsection{Optical and NIR Upper Limits}

The brightness of scattered light from circumstellar material can be estimated from

$$
R_{\lambda, r}=\left(\tau_{\perp} / 4 \pi\right)\left(d_{\mathrm{pc}}^{2} / r_{\mathrm{AU}}^{2}\right)(P \phi(\gamma) / \cos (i))
$$

in which $R$ is the ratio, at a given wavelength and position, of circumstellar surface brightness per square arcsec to the star's brightness; $\tau_{\perp}$ is the perpendicular optical depth of the circumstellar material; $d_{\mathrm{pc}}$ is the system distance from the observer; $r_{\mathrm{AU}}$ is the distance of the material from the star; $P$ is the geometric albedo; $\phi(\gamma)$ is the phase function value for the stardust-observer angle $\gamma$; and $\cos (i)$ is the inclination of the disk to the plane of the sky, $i=0$ representing face-on presentation. A value of $\cos (i) \sim 1$ can be used for the nearly face-on $\epsilon$ Eri disk. A value $\phi(\gamma) \sim 0.5$ can be assumed for particles much larger than the wavelength of interest in an approximately face-on disk.

Proffitt et al. (2004) used the STIS spectrographic camera on $H S T$ to search for scattered light from the sub-mm ring region. They set an upper limit of $m_{\text {STIS }} \gtrsim+25$ for circumstellar light 
at $r \sim 55 \mathrm{AU}\left(17^{\prime \prime}\right)$ in a wide bandpass with $\lambda_{\text {eff }} \sim 0.6 \mu \mathrm{m}$. Assuming an albedo of $P=0.1$ for the model large grains, which have the highest optical depth in the sub-mm ring region (Figure 6(a)), yields a predicted scattered light surface brightness at $55 \mathrm{AU}$ almost exactly equal to the HST/STIS limit. Assuming, instead, an albedo of 0.9 (possible for fresh pure ice surfaces) would yield an optical surface brightness that is almost 2.5 mag brighter than the limit. In comparison, the model of the second warm belt $\left(r \sim 20 \mathrm{AU} \sim 6^{\prime \prime}\right)$ predicts an optical surface brightness of $m_{V} \sim+21.7$ per square arcsec assuming $P=0.1$ and radial width $\Delta r=2 \mathrm{AU}$, brightness scaling inversely with $\Delta r$.

Di Folco et al. (2007) reported NIR ( $K^{\prime}$ band, $2.1 \mu \mathrm{m}$ ) interferometric observations of $\epsilon$ Eri and $\tau$ Ceti with the CHARA array. They resolved the stellar diameters in both cases and detected circumstellar emission near $\tau$ Ceti but set an upper limit of $0.6 \%$ of the photosphere for emission within $1^{\prime \prime}(3 \mathrm{AU})$ of $\epsilon$ Eri. The present model's innermost belt predicts a total scattered light brightness of approximately 0.03 times the $\mathrm{Di}$ Folco et al. (2007) upper limit if the grain NIR albedo is assumed to be 0.1 . The direct thermal emission at the $K^{\prime}$ band from that belt, in fact from the entire disk, would be many orders of magnitude fainter than the scattered light brightness.

\subsubsection{SED Models}

Dent et al. (2000), Li et al. (2003; hereafter LLB03), and Sheret et al. (2004; hereafter SDW04) combined pre-Spitzer broadband photometry and sub-mm map data to produce models of the $\epsilon$ Eri disk with specific grain spatial distributions, size distributions, and compositions. Given the limited data available to constrain them, those models are consistent with the model presented in Section 4 of this paper based on higher-precision observations over a much broader wavelength range.

Dent et al. (2000) assumed nonporous grains, with a simplified emissivity law determined only by particle size without mineralogical features. Their model requires material as close as $10 \mathrm{AU}$ from the star with $\sim 10 \%$ of the surface density in the main ring. LLB03 and SDW04 calculated models assuming real grain mineralogies, including a range of porosities (fraction of silicate grain volume occupied by vacuum or ice) ranging from $p=0$ to 0.9 . SDW04 included their own sub-mm photometric data regarding $\epsilon$ Eri to supplement published maps. LLB03's best-fit model involved highly porous $(p=0.9)$ grains, whereas SDW04's suite of models favored nonporous (solid) grains. The present model assumes nonporous grains and qualitatively agrees with the SDW04 result that the grain size distribution cannot extend down to $1 \mu \mathrm{m}$ without producing too much short- $\lambda$ emission. SDW04 remarked that the lack of $\mu \mathrm{m}$-scale grains is a puzzle, given the inability of $\epsilon$ Eri's radiation field to eject grains of that size. Radiation pressure and corpuscular wind effects are further discussed in Section 5.3.

The total circumstellar grain mass derived by LLB03 was $\sim 7 \times 10^{-3} M_{\oplus} \sim 0.6 M_{\text {Luna }}$, compared with $0.1 M_{\text {Luna }}$ in SDW04's model and $0.4 M_{\text {Luna }}$ in the current model. The differences between these mass estimates result from differing assumptions about the particle properties and size ranges, since each group approximately matches the same system fluxes, requiring similar total particle radiating areas.

\subsection{Particle Dynamics}

\subsubsection{Grain Ejection via Wind Versus Radiation Pressure}

Small grains are subject to radiation pressure (e.g., Burns et al. 1979) and corpuscular winds (e.g., Plavchan et al. 2005) that tend to remove them from their source region, either rapidly outward by direct ejection for particles smaller than the "blowout" size or, for larger particles, slowly inward toward encounters with planets or sublimation near the star. Grains also collide destructively. Competition between rates of destructive processes versus the rate of grain resupply from larger parent bodies determines a debris disk's equilibrium density and also whether there is net mass flow outward or inward from the grain source (e.g., Wyatt 2005; Meyer et al. 2007). These processes will be considered in this and the following subsection in view of the $\epsilon$ Eri system's characteristics. Porous and nonspherical grains would be more strongly affected by radiation and wind than the spherical and nonporous grains assumed here, so destruction and drift timescales calculated below should be considered upper limits.

The direct (radial) radiation pressure (force per area) opposing gravitational attraction between a star and grain is

$$
P_{r}(\text { radiation })=L_{*} / 4 \pi r^{2} c,
$$

whereas the direct wind pressure is

$$
P_{r}(\text { wind })=v_{\text {wind }}\left(d M_{*} / d t\right) / 4 \pi r^{2} .
$$

The wind mass-loss rate for $\epsilon$ Eri has been determined to be $\sim 3-4 \times 10^{10} \mathrm{~kg} \mathrm{~s}^{-1}$ (Stevens 2005; Wood et al. 2005), about $20-30 \times$ the solar value. Assuming conservatively that young, chromospherically active $\epsilon$ Eri has the same wind terminal speed of $\sim 400 \mathrm{~km} \mathrm{~s}^{-1}$ as the Sun's, radial wind pressure is negligible in the $\epsilon$ Eri system-less than $5 \%$ as strong as radiation pressure. The star's mass/luminosity ratio is low enough that solid silicate-density particles should be stable against direct ejection down to sizes of a few $\times 0.1 \mu \mathrm{m}$.

This analysis indicates that (1) the model grain size lower limits of a few microns in the sub-mm ring and inner unresolved belts are not defined by radiation pressure or neutral wind "blowout" and (2) radiation and wind pressures in this system are too weak to move substantial amounts of material outward, for example from the sub-mm ring into the halo.

\subsubsection{P-R and Wind Azimuthal Drag Versus Collisions}

The following two equations compare the azimuthal pressure $P_{\theta}$ on orbiting grains, respectively, due to radiation (P-R drag) and a neutral wind:

$$
\begin{aligned}
& P_{\theta}(\text { radiation })=v_{\mathrm{orb}} L_{*} / 4 \pi r^{2} c^{2}, \\
& P_{\theta}(\text { wind })=v_{\mathrm{orb}}\left(d M_{*} / d t\right) / 4 \pi r^{2} .
\end{aligned}
$$

Using the values given above for wind mass and speed, the radial drift rate for particles around $\epsilon$ Eri is enhanced by a factor of about 20 relative to the purely radiation-driven $\mathrm{P}-\mathrm{R}$ drag rate (numerically similar, by coincidence, to the $\epsilon$ Eri wind mass loss rate relative to solar).

The timescale for grain-grain mutual collisions is

$$
t_{\text {coll }} \sim P_{\text {orb }} / 8 \tau_{\perp},
$$


in which $P_{\text {orb }}$ is the orbit period and $\tau_{\perp}$ is the perpendicular optical depth. Cross-velocities as low as a few hundred meters per second are sufficient to destroy small silicate and ice grains. The solar system's asteroid and Kuiper belts both have inclination and eccentricity ranges more than large enough to result in routinely shattering collisions, which may also be assumed to pertain to the $\epsilon$ Eri disk.

At the inner edge of the sub-mm ring, $r=35 \mathrm{AU}$, where the $\mathrm{P}-\mathrm{R}$ drag is most competitive with collisions, the collision timescale for the large $(a \sim 135 \mu \mathrm{m})$ grains is $t_{\text {coll }} \sim 2 \times 10^{5} \mathrm{yr}$, whereas the wind-drag timescale for inward drift by $\Delta r=1 \mathrm{AU}$ is $\sim 8 \times 10^{5} \mathrm{yr}$. Thus, even when enhanced by wind, virtually all the large icy grains should be destroyed by collisions in situ and not be able to drift inward to fill the central void. For the small grains $(a \sim 15 \mu \mathrm{m})$, on the other hand, the collision destruction timescale (including the cross-sectional area of both small and large grains as targets) is $\sim 1.5 \times 10^{5} \mathrm{yr}$, approximately the same as the wind-drag timescale for these particles to drift 1 AU. Given that the collision process is statistical, for example $\sim 1 \%$ of grains can be expected to survive $5 t_{\text {coll }}$, some fraction of the small grain population could travel inward from the sub-mm ring into the central void before colliding with other grains.

At the inner and outer edges of the halo, $r=90$ and $110 \mathrm{AU}$, respectively, the collision timescale for the small grains is also comparable to the $\Delta r=1 \mathrm{AU}$ wind-drag drift time. Thus, if grains are produced in the halo region, they would tend to stay in place or flow inward toward the sub-mm ring, rather than outward.

\subsubsection{Collisional Scattering}

Is there a mechanism other than radiation or wind pressure that could move material from the sub-mm ring to the outer halo, or does the presence of significant material in the halo require a particle source (presumably collisions of planetesimals) at $r \gtrsim 90 \mathrm{AU}$ ? In fact, scattering of material into new orbits after collisions could suffice.

The vis-viva equation expresses the orbit properties resulting from velocity other than circular orbit velocity at a given location,

$$
v^{2}=G M_{*}\left(2 / r-1 / a_{\mathrm{orb}}\right),
$$

in which $v$ is the speed at radius $r$ and $a_{\text {orb }}$ is the orbit semimajor axis.

Under what conditions would grain collisions in the main, heavily-populated ring be able to supply material to the outer halo? If grains or parent bodies collide at $r$ with average crossspeeds of $\xi \times v_{\text {circ }}$, corresponding to relative orbit inclinations of $\operatorname{order} \delta i \sim \pm \xi \mathrm{rad}$ and/or eccentricity ranges of order $\delta e \sim \xi / 2$, significant numbers of fragments will leave the collision with $\delta v \geqslant \xi v$ relative to the motion of the colliding bodies' center of mass. Collisions at $r \sim 90 \mathrm{AU}$ with $\xi=0.05$ would send some fragments into new orbits reaching to $110 \mathrm{AU}$, the outer edge of the halo. Therefore, the extended halo observed at 70 and $160 \mu \mathrm{m}$ could be the result of collisions among planetesimals and fragments moving with modest departure from circular planar motion in the sub-mm ring. If collisions are, in fact, the mechanism spreading material from the ring into the halo, the lack of material beyond $110 \mathrm{AU}$ could indicate an upper limit on inclinations and eccentricities in the ring.

\subsubsection{Particle Dynamics Summary}

Radiation plus wind pressure should not be able to eject solid silicate-density particles larger than the submicron size from the $\epsilon$ Eri system, but the disk model has a deficit of particles smaller than $a \sim 6 \mu \mathrm{m}$ in the sub-mm ring. Consideration of particle destruction timescales suggests that a time span of order $10^{5}$ years may have elapsed since the last significant injection of small particles. The small grain population has a spatial distribution, indicating overall control by azimuthal drag. Some small grains near the inner edge of the sub-mm ring should be able to drift into the central void faster than their collision destruction timescale. The fact that this does not appear to be happening may indicate the presence of a barrier planet preventing inward drift (Section 5.4).

The large grains in the sub-mm ring have collision timescales much shorter than drift timescales, so they are seen near their locations of creation, and their radial mass density profile may track that of the planetesimal parent bodies.

\subsection{Planets Around $\epsilon$ Eri}

Radial velocity measurements of $\epsilon$ Eri have suggested the presence of a planetary companion, hereafter designated Planet A, with $M \sin i=0.86 M_{\mathrm{Jup}}$ and an orbit period of about $6.9 \mathrm{yr}$ (Hatzes et al. 2000 and references therein). The corresponding orbital semimajor axis is $3.4 \mathrm{AU}$, near the location inferred for the innermost warm dust belt.

The eccentricity of $0.70 \pm 0.04$ reported for the $3.4 \mathrm{AU}$ planet by Benedict et al. (2006) would correspond to periastron and apoastron distances of 1.0 and $5.8 \mathrm{AU}$, respectively. A giant planet with this orbit would quickly clear that region not only of dust particles but also the parent planetesimal belt needed to resupply them, inconsistent with the dust distribution implied by the Spitzer observations. The disk's 70, 160, and $350 \mu \mathrm{m}$ aspect ratios and position angles reported here differ noticeably from values of those quantities determined from the $850 \mu \mathrm{m}$ map, calling into question the system inclination value used to combine the astrometric- and radial-velocity data. Butler et al. (2006) reported a different orbital eccentricity of $0.25 \pm$ 0.23 , which could allow the planet to orbit entirely outside the innermost dust belt.

As discussed in Section 5.3, some of the small grains in the sub-mm ring should be able to drift into the central void and begin to fill it on timescales of $10^{6}-10^{7}$ years. A mechanism is needed to prevent grains from moving inward past $35 \mathrm{AU}$. Liou \& Zook (1999) and Moro-Martin et al. (2005) modeled the ability of massive planets to define the inner edges of Kuiper belt-like dust rings. A hypothetical planet orbiting at the edge of the main $\epsilon$ Eri sub-mm ring is here designated Planet C. The gravitational influence of a planet near or in the ring has also been proposed (Liou \& Zook 1999; Quillen \& Thorndike 2002; Greaves et al. 2005) as the cause of ring density enhancements corresponding to surface brightness features reported in sub-mm imaging that may or may not be real (Section 5.2.1).

Confinement of a narrow debris belt at $r \sim 20$ AU could indicate the presence of a third planet, here designated Planet B. Deller \& Maddison (2005) calculated dynamical models of the $\epsilon$ Eri system including effects of planetary masses, radiation pressure, and stellar wind, and found that a planet orbiting at the inner edge of the sub-mm ring would not by itself be able to define the ring's edge. Rather, the effect of an additional Jovian-mass planet with a semimajor axis $a \simeq 20 \mathrm{AU}$ is needed. Greaves et al. (2005) proposed that the mass controlling some of the possible sub-mm ring features may be located well inside the ring, at $r \sim 24 \mathrm{AU}$, based on the orbital period suggested by apparent motion of these features over 7 years. 
Summarizing all the observations and models bearing on the presence of planets around $\epsilon$ Eri, there may be indications of three different planets in the system. Planet A: the longsuspected but still unconfirmed Jovian-mass planet with $a_{\text {orb }}=$ 3.4 AU that may be associated with the innermost warm debris belt detected by Spitzer. Planet B: also perhaps Jovian-mass, associated with the second warm debris belt at $r \sim 20 \mathrm{AU}$ inferred in this paper, also possibly helping define the sub-mm ring inner edge via 2:1 resonance, keeping the zone between 20 and 35 AU clear. Note that a $1 M_{\text {Jup }}$ planet with an age of 0.85 Gyr located 6"from $\epsilon$ Eri would not have been seen by Spitzer but could be detected by James Webb Space Telescope $(J W S T)$. Planet C: located at $r \sim 35 \mathrm{AU}$, with mass less than few $\times 0.1 M_{\text {Jup }}$ according to dynamical models, preventing small grains from drifting inward past the inner edge of the sub-mm ring.

\subsection{Simple Collisional-Evolutionary Model}

A dynamical model of the $\epsilon$ Eri sub-mm ring was calculated assuming equilibrium between collisional production of grains and removal by $\mathrm{P}-\mathrm{R}$ plus corpuscular wind drag, based on previous simple dynamical models of the solar system's Kuiper belt (Backman et al. 1995) and solar-type circumstellar debris disks observed by Spitzer (Meyer et al. 2007). This model indicates that the sub-mm ring can be maintained by collisions of $11 M_{\oplus}$ of parent bodies with assumed diameters of $10 \mathrm{~km}$ and material densities of $1000 \mathrm{~kg} \mathrm{~m}^{-3}$. This agrees with estimates made by Sheret et al. (2004) and Greaves et al. (2005) using similar assumptions. Thus, the $\epsilon$ Eri system as observed can be in quasi-equilibrium.

Spitzer observations of Vega revealed a FIR disk extending across the position of a sub-mm ring into an outer halo (Su et al. $2005)$, similar to the $\epsilon$ Eri system. However, the Vega disk's properties require recent injection of circumstellar material to explain the abundance of short-lived particles. The main difference between the two systems is that $\epsilon$ Eri's luminosity (including wind) is low relative to Vega's, so there is no direct ejection of grains, P-R-plus-wind azimuthal drag rates are lower, and equilibrium resupply by continuous minor collisions can be effective.

A simple evolutionary model that estimates loss of belt mass via destruction of planetesimal parent bodies, with subsequent removal of fragments by the dynamical processes listed above, was described and applied to the solar system's Kuiper belt, asteroid belt, and extrasolar analogs by Meyer et al. (2007). The same model, tuned to the properties of the $\epsilon$ Eri system, predicts that for an initial mass of planetesimal parent bodies in the range 15-30 $M_{\oplus}$, similar to the inferred original mass of the Kuiper belt (e.g., Stern \& Colwell 1997), about $11 M_{\oplus}$ would remain at a system age of 0.85 Gyr. In other words, $\epsilon$ Eri's current debris disk is similar in total mass to what the solar system's Kuiper belt mass would have been at the age of $\epsilon$ Eri had there not been a prior Late Heavy Bombardment (LHB) clearing event (e.g., Levison et al. 2007).

Collisional evolution is dissipative, eventually converging on a planetesimal population that has a timescale for further collisions proportional to the system age, nearly independent of the initial conditions, if there are no external effects. However, to transform the young solar system's Kuiper belt or $\epsilon$ Eri's debris disk into a low-mass system like the present-day Kuiper belt, with at most a few $\times 0.1 M_{\oplus}$ at age $4.5 \mathrm{Gyr}$ (Luu \& Jewitt 2002), requires additional mass-reduction processes beyond quasisteady state collisional grinding and debris removal (Meyer et al. 2007 and references therein). For example, in our solar system, the LHB event, possibly driven by a late episode of planet migration, may have destroyed most of the Kuiper belt's mass relatively suddenly, and similar events may happen in other systems with ages of a Gyr or more (Levison et al. 2007 and references therein). Alternatively, a process that is continually at work in our solar system, planetary perturbations pulling planetesimals out of the Kuiper belt, could, with the help of collisional grinding, transform $\epsilon$ Eri's $11 M_{\oplus}$ disk at $t=0.85 \mathrm{Gyr}$ into the solar system's $0.1 M_{\oplus}$ Kuiper belt at $4.5 \mathrm{Gyr}$ if the perturbative removal process supplementing collisions were to operate with an exponential timescale of about 1.5 Gyr. Note that $\tau$ Ceti's debris disk has an inferred mass of $1.2 M_{\oplus}$ at an age of $8 \mathrm{Gyr}$, so it may not have had a major disruptive episode (Greaves et al. 2004).

\section{SUMMARY}

The planetary debris disk around $\epsilon$ Eri was observed using Spitzer's IRAC and MIPS cameras, Spitzer's IRS and MIPS SED-mode spectrophotometers, and the SHARC II bolometer array on the CSO submillimeter telescope.

The slight $25 \mu \mathrm{m}$ excess detected by IRAS is photometrically confirmed by the Spitzer IRS and MIPS data but not resolved in the $24 \mu \mathrm{m}$ image. The CSO $350 \mu \mathrm{m}$ map confirms the sub-mm ring at $r \sim 11-28^{\prime \prime}(35-90 \mathrm{AU})$, previously observed at 450 and $850 \mu \mathrm{m}$, about $2 \times$ the width of our solar system's Kuiper belt. Analyses of the 70 and $160 \mu \mathrm{m}$ images reveal emission extending from within a few arcsec of the star outward to $r \sim 34^{\prime \prime}(110 \mathrm{AU})$, both inside and outside the sub-mm ring. The Spitzer 70 and $160 \mu \mathrm{m}$ images do not have enhanced surface brightness at the position of the ring. The $350 \mu \mathrm{m}$ surface brightness noticeably varies with azimuth around the ring but the locations of the brightest features do not correspond between $350 \mu \mathrm{m}$ data sets reduced separately, or with the brightest features in the longer-wavelength maps, calling into question the reality of the sub-mm ring azimuthal asymmetries.

The overall system SED is complex, with significant excess relative to the photosphere beginning at $\lambda \sim 15 \mu \mathrm{m}$. The excess rises rapidly to $20 \mu \mathrm{m}$, flattens from 20 to $30 \mu \mathrm{m}$, rises rapidly again from $30 \mu \mathrm{m}$ to a peak between 70 and $100 \mu \mathrm{m}$, and then decreases toward longer wavelengths.

A model of the disk, constrained by all the available data, combines two populations of material in the resolved disk: (1) large (radii $a \sim 100-200 \mu \mathrm{m}$ ) solid grains in the sub-mm ring, which have high emissivity at sub-mm wavelengths but low emissivity at 70 and $160 \mu \mathrm{m}$, consistent with $\mathrm{H}_{2} \mathrm{O}$ ice composition, plus (2) smaller ( $a \sim 6-23 \mu \mathrm{m})$ solid grains in both the sub-mm ring and its exterior "halo" $(r=25-34$ ", 90-110 AU) which have high emissivity in the FIR and low emissivity at sub-mm wavelengths, consistent with silicate composition. The mid-IR and FIR spectrophotometry and radial source profiles imply two additional narrow belts of material, one at $r \sim 3 \mathrm{AU}\left(1^{\prime \prime}\right)$ with a weak $20 \mu \mathrm{m}$ silicate emission feature and the other at $r \sim 20 \mathrm{AU}\left(6^{\prime \prime}\right)$ of undetermined, but possibly silicate, composition.

The grains in the halo may be spread there from the ring region via the effect of collisions. Definition of the inner edge of the sub-mm ring, and confinement of the two rings of material closer to the star, probably requires gravitational influences of three planets. A dynamical model of the disk indicates that the observed populations of grains in the sub-mm ring and outer halo can be supplied in equilibrium by collisions of about $11 M_{\oplus}$ of $\mathrm{km}$-scale parent bodies located in the sub-mm ring. 
This paper is based in part on observations made with the Spitzer Space Telescope that is operated by the Jet Propulsion Laboratory, California Institute of Technology, under NASA contract 1407, and in part on observations from the CSO. Support for this work was provided by NASA through awards issued by JPL/Caltech, including subcontracts 1278243 and 1255094, respectively, to the SETI Institute and the University of Arizona, and also by NASA grant NNG05GI81G. Analyses in this paper made use of the SIMBAD database operating at CDS, Strasbourg, France.

We are grateful to J. Morrison and M. Blaylock, University of Arizona, for help with MIPS image data processing, and P. Smith, University of Arizona, for guidance in MIPS SEDmode data processing. We thank P. Plavchan and E. Becklin for helpful discussions.

\section{REFERENCES}

Altenhoff, W. J., Bertoldi, F., Menten, K. M., Sievers, A., Thum, C., \& Kreysa, E. 2002, A\&A, 391, 353

Aumann, H. H. 1985, PASP, 97, 885

Backman, D. 2004, in ASP Conf. Ser. 324, Debris Disks and the Formation of Planets: A Symposium in Memory of Fred Gillett, ed. L. Caroff, L. J. Moon, D. Backman, \& E. Praton (San Francisco, CA: ASP), 9

Backman, D., \& Paresce, F. 1993, in Protostars and Planets III, ed. E. H. Levy \& J. I. Lunine (Tucson, AZ: Univ. Arizona Press), 1253

Backman, D. E., Dasgupta, A., \& Stencel, R. E. 1995, ApJ, 450, L35

Barnes, S. A. 2007, ApJ, 669, 1167

Benedict, G. F., et al. 2006, AJ, 132, 2206

Burns, J. A., Lamy, P. L., \& Soter, S. 1979, Icarus, 40, 1

Butler, R. P., et al. 2006, ApJ, 646, 505

Campbell, B. W., Walker, G. A. H., \& Yang, S. 1988, ApJ, 331, 902

Chen, C. H., \& Jura, M. 2001, ApJ, 560, L171

Chen, C. H., et al. 2006, ApJS, 166, 351

Chen, C. H., et al. 2007, ApJ, 666, 466

Cohen, M., Megeath, S. T., Hammerseley, P. L., Martín-Luis, F., \& Stauffer, J. 2003, AJ, 125, 2645

Das, S. R., \& Backman, D. E. 1992, BAAS, 24, 1228

Deller, A. T., \& Maddison, S. T. 2005, ApJ, 625, 398

Dent, W. R. F., Walker, H. J., Holland, W. S., \& Greaves, J. S. 2000, MNRAS, 314, 702

Di Folco, E., Thévenin, F., Kervella, P., Domiciano de Souza, A., Coudé du Foresto, V., Sńgransan, D., \& Morel, P. 2004, A\&A, 426, 601

Di Folco, E., et al. 2007, A\&A, 475, 234

Dowell, C. D., et al. 2003, in Proc. SPIE 4855, Millimeter and Submillimeter Detectors for Astronomy, ed. T. G. Phillips \& J. Zmuidzinas (Bellingham, WA: SPIE), 73

Engelbracht, C. W., et al. 2007, PASP, 119, 994

Fazio, G. G., et al. 2004, ApJS, 154, 10

Flower, P. J. 1996, ApJ, 469, 355

Gillett, F. C. 1984, invited oral presentation at Protostars and Planets II

Gillett, F. C. 1986, in Proc. the First Infra-Red Astronomical Satellite Conference, Light on dark matter (Dordrecht: Reidel Publishing), 61

Gordon, K. D., et al. 2005, PASP, 117, 503

Gordon, K. D., et al. 2007, PASP, 119, 1019

Greaves, J., et al. 1998, ApJ, 506, 133
Greaves, J., et al. 2005, ApJ, 619, 187

Greaves, J. S., Wyatt, M. C., Holland, W. S., \& Dent, W. R. F. 2004, MNRAS, 351, L54

Hatzes, A., et al. 2000, ApJ, 544, L145

Henry, T. J., Soderblom, D. R., Donahue, R. A., \& Baliunas, S. L. 1996, AJ, 111,439

Houck, J. R., et al. 2004, ApJS, 154, 18

Johnson, H. L. 1986, ARA\&A, 4, 193

Koornneef, J. 1983a, A\&AS, 51, 489

Koornneef, J. 1983b, A\&A, 128, 84

Krist, J. E. 2006, Spitzer Tiny TIM User's Guide Version 2.0

Lagrange, A.-M., Backman, D. E., \& Artymowicz, P. 2000, in Protostars and Planets IV, ed. V. Mannings, A. P. Boss, \& S. S. Russell (Tucson, AZ: Univ. Arizona Press), 639

Levison, H. F., Morbidelli, A., Gomes, R., \& Backman, D. 2007, in Protostars and Planets V, ed. B. Reipurth, D. Jewitt, \& K. Kiel (Tucson, AZ: Univ. Arizona Press), 669

Li, A., Lunine, J. I., \& Bendo, G. J. 2003, ApJ, 598, L51

Liou, J.-C., \& Zook, H. A. 1999, AJ, 118, 580

Lisse, C. M., Chen, C. H., Wyatt, M. C., \& Morlok, A. 2008, ApJ, 673,1106

Luu, J. X., \& Jewitt, D. C. 2002, ARAA, 40, 63

Marengo, M., Megeath, S. T., Fazio, G. G., Stapelfeldt, K. R., Werner, M. W., \& Backman, D. E. 2006, ApJ, 647, 1437

Mendoza, E. E., Gomez, V. T., \& Gonzalez, S. 1978, AJ, 83, 606

Meyer, M. R., Backman, D. E., Weinberger, A. J., \& Wyatt, M. C. 2007, in Protostars and Planets V, ed. B. Reipurth, D. Jewitt, \& K. Kiel (Tucson, AZ: Univ. Arizona Press), 573

Moran, S. M., Kuchner, M. J., \& Holman, M. J. 2004, ApJ, 612, 1163

Moro-Martin, A., Wolf, S., \& Malhotra, R. 2005, ApJ, 621, 1079

Ozernoy, L. M., Gorkavyi, N. N., Mather, J. C., \& Taidakova, T. A. 2000, ApJ, 537, 147

Plavchan, P., Jura, M., \& Lipscy, S. J. 2005, ApJ, 631, 1161

Poulton, C. J., Greaves, J. S., \& Cameron, A. C. 2006, MNRAS, 372, 53

Proffitt, C. R., et al. 2004, ApJ, 612, 481

Quillen, A. C., \& Thorndike, S. 2002, ApJ, 578, L149

Rieke, G., et al. 2004, ApJS, 154, 25

Schuster, M., Marengo, M., \& Patten, B. 2006, SPIE Meeting (Orlando, FL: SPIE), 6270, 627020

Schütz, O., Nielbock, M., Wolf, S., Henning, Th., \& Els, S. 2004, A\&A, 414, L9

Sheret, I., Dent, W. R. F., \& Wyatt, M. C. 2004, MNRAS, 348, 1282

Song, I., Caillault, J.-P., Barrado y Navascués, D., Stauffer, J. R., \& Randich, S 2000, ApJ, 533, L41

Stansberry, J. A., et al. 2007, PASP, 119, 1038

Stapelfeldt, K. R., et al. 2004, ApJS, 154, 458

Stern, S. A., \& Colwell, J. E. 1997, ApJ, 490, 879

Stevens, I. R. 2005, MNRAS, 356, 1053

Su, K. Y., Rieke, G. H., Stapelfeldt, K. R., Smith, P. S., Bryden, G., Chen, C. H., \& Trilling, D. E. 2008, ApJ, 679, 125

Su, K. Y., et al. 2005, ApJ, 628, 487

Su, K. Y., et al. 2006, ApJ, 653, 675

Trilling, D. E., et al. 2008, ApJ, 674, 1086

Walker, H. J., \& Heinrichsen, I. 2000, Icarus, 143, 147

Wolf, S., \& Hillenbrand, L. A. 2005, Comp. Phys. Commun., 171,208

Wood, B. E., Müller, H.-R., Zank, G. P., Linsky, J. L., \& Redfield, S. 2005, ApJ, 628, L143

Wyatt, M. C. 2005, A\&A, 433, 1007

Wyatt, M. C. 2006, ApJ, 639, 1153 